\title{
Efficient Construction of Unified Continuous and Discontinuous Galerkin Formulations for the 3D Euler Equations
}

\author{
Daniel S. Abdi ${ }^{\mathrm{a}, *}$, Francis X. Giraldo ${ }^{\mathrm{a}, *}$ \\ ${ }^{a}$ Department of Applied Mathematics, Naval Postgraduate School, Monterey, CA, USA
}

\begin{abstract}
A unified approach for the numerical solution of the 3D hyperbolic Euler equations using high order methods, namely continuous Galerkin (CG) and discontinuous Galerkin (DG) methods, is presented. First, we examine how classical CG that uses a global storage scheme can be constructed within the DG framework using constraint imposition techniques commonly used in the finite element literature. Then, we implement and test a simplified version in the Non-hydrostatic Unified Model of the Atmosphere (NUMA) for the case of explicit time integration and a diagonal mass matrix. Constructing CG within the DG framework allows CG to benefit from the desirable properties of DG such as, easier $h p$-refinement, better stability etc. Moreover, this representation allows for regional mixing of CG and DG depending on the flow regime in an area. The different flavors of CG and DG in the unified implementation are then tested for accuracy and performance using a suite of benchmark problems representative of cloud-resolving scale, meso-scale and global-scale atmospheric dynamics. The value of our unified approach is that we are able to show how to carry both CG and DG methods within the same code and also offer a simple recipe for modifying an existing $\mathrm{CG}$ code to $\mathrm{DG}$ and vice versa.
\end{abstract}

Keywords: NUMA, continuous Galerkin, discontinuous Galerkin

\section{Introduction}

Current global Numerical Weather Prediction (NWP) models operate with a maximum resolution of $10-20 \mathrm{~km}$. At this scale of resolution, both hydrostatic and non-hydrostatic models may be used, however, various agencies such as the National Oceanic and Atmospheric Administration (NOAA) have set a goal of $3 \mathrm{~km}$ resolution for an operational NWP model within the next decade [33]. Therefore, it is of paramount importance that state-of-the-art NWP models

*Department of Applied Mathematics, Naval Postgraduate School, Monterey, CA, USA, Email: dsabdi@nps.edu,fxgirald@nps.edu

Preprint submitted to TBD

April 23, 2016

(C) 2016. This manuscript version is made available under the Elsevier user license http://www.elsevier.com/open-access/userlicense/1.0/ 
account for non-hydrostatic effects with increased resolution. This work is conducted using the Non-hydrostatic Unified Model of the Atmosphere (NUMA) [14] which we believe is well placed to meet this goal. NUMA has the following features: a) non-hydrostatic, b) high-order element based Galerkin (EBG) methods for spatial discretization, c) unified regional and global NWP, d) static/dynamic Adaptive Mesh Refinement (AMR), e) high-order explicit, implicitexplicit (IMEX) and fully implicit temporal discretization, f) scalable to millions of CPUs [29] and thousands of GPUs [39]. Porting NUMA to next generation many-core architectures is currently an ongoing effort. So far we have managed to accelerate NUMA by upto 240x using one NVIDIA K20X GPU relative to one core of an AMD CPU. Also, NUMA currently achieves a weak scaling efficiency of about $90 \%$ using 16384 GPUs on the Titan supercomputer, the second fastest supercomputer in the world as of 2015 [9].

The focus of this paper is on the unification of the high-order spatial discretization methods used in NUMA, namely continuous Galerkin (CG) and discontinuous Galerkin (DG), for the solution of the hyperbolic Euler equations. Within the realm of atmospheric modeling, the first use of high order CG appeared in $[17,12]$ and that of high order DG methods in $[14,30]$. CG has been the most popular choice for solving Partial Differential Equations (PDEs) in solid mechanics, however, DG is becoming more popular in the computational fluid dynamics field due to several desirable properties it possesses [31]. Unified analysis of different spatial discretization schemes helps to understand and compare methods which at first seem very different. As such, there have been efforts in the past to unify different flavors of CG and DG. Unified analysis of several variants of DG methods for elliptic problems can be found in [1] and that of hybridized CG and DG methods in [7].

In this work, we wish to construct CG using the local element wise storage scheme of DG so that CG can immediately benefit from several desirable properties of DG. We discuss briefly some of the advantages of DG compared to CG. Adaptive grid methods, $h$ - and $p$-refinement, are easier to implement and more flexible in DG than CG; although it is possible to use $h$ and $p$ refinement with CG methods (see $[25,36,28]$ for CG with $h$-refined AMR). For advection dominated problems, in which the first order advection term is larger than the second-order diffusive term, i.e. Peclet number $P e>1$, the classical CG method can produce inaccurate results due to spurious oscillations. In contrast, DG can be significantly more stable through its use of an upwind-biased numerical flux. Thus, DG is naturally more suited for capturing strong gradients (shocks) in solutions than CG without showing spurious oscillations. One way to stabilize CG is to add artificial diffusion to increase the influence of the second-order diffusive term to the level of the first-order advective term. We use this stabilization method for both CG and DG simulations conducted in this work. The locally conservative nature of DG also makes it more suited for conservation laws just like its popular low-order counterpart, the Finite Volume Method (FVM). Parallel implementation of DG on a cluster of CPUs is often more efficient and easier than that of CG [21, 19]. DG is also more efficient on Graphic Processing Units (GPUs) because most of the computation is local 


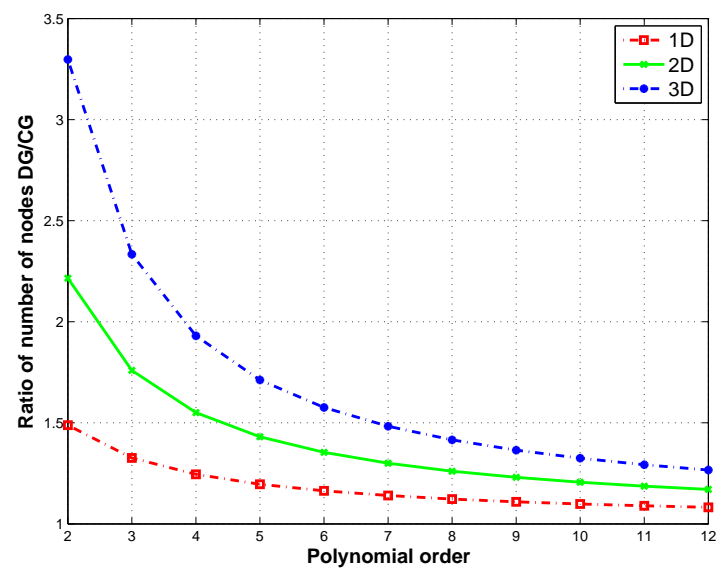

Figure 1: Ratio of number of nodes in DG vs CG for the range of polynomial orders often used in NUMA. The plots are made for 64 tensor-product hexahedral elements in $1 \mathrm{D}, 2 \mathrm{D}$ and $3 \mathrm{D}$ domain. For the highest polynomial order, the overhead of DG is 1.08 in 1D, 1.17 in 2D and 1.26 in $3 \mathrm{D}$.

to each element [24]. Finally, the unification would allow for a regional mixing of CG and DG, such that CG is used in parts of the domain where the flow is smooth, and DG in areas where the flow is non-smooth. Coupled CG and DG methods have been investigated in the past in $[8,6]$. In [8], a local discontinuous Galerkin method is coupled with CG, with transmission conditions used where CG and DG regions meet. In [6], the stability of a coupled CG and DG method is studied. The authors note that, even though a coupled CG and DG method without using additional transmission conditions is more natural, it can result in under- and overshoots at the interfaces of CG and DG regions. In this work, we do not impose transmission conditions, other than applying the inherent coupling mechanisms of CG and DG. Usually this will not cause a problem if the non-smooth region is entirely contained within the DG region. We should note here that there are other ways of mixing CG and DG for numerical solution of PDEs, for instance, using CG for second-order terms and DG for first order terms, however, we will not pursue this approach in this paper.

The single major advantage of $C G$ is that its classical implementation results in less degrees of freedom than that of DG which uses duplicate nodes on faces shared by two elements to model discontinuities better. Nguyen and Peraire [31] give an example with six linear and cubic tetrahedral elements, for which the ratio of nodes between DG and CG is 24 and 5 respectively. However, this criticism is not valid for high-order tensor-product hexahedral elements. For example, using $4^{3}=64$ hexahedral elements and cubic polynomials, the ratio of the number of nodes between DG and CG is 1.86 , and using $6^{\text {th }}$ order polynomials the ratio goes down to 1.3. As the degree of the polynomial increases, the ratio approaches 1 . In NUMA, we routinely use high-order hexahedral elements therefore opting to conduct CG simulations within the DG framework will not 
result in significant overhead. The ratio of the number of nodes of DG to CG is shown in Fig. 1 for the range of polynomial orders often used in NUMA. We should also note here that there exist DG methods, such as Hybridizable Discontinuous Galerkin (HDG), that are computationally competitive with CG at moderate polynomial orders [23], although a thorough analysis of the computational cost of HDG is required.

In this paper, we shall first present how we can construct CG within the DG framework using a local element wise storage scheme; it is not possible to do the converse, namely DG within the classical CG framework that uses a global storage scheme, which implicitly satisfies continuity requirement at shared nodes. Mathematically speaking, the main difference between CG and DG lies in the definition of the finite dimensional vector spaces used to define the set of basis functions and, hence, the solutions. CG uses a relatively restrictive $H^{1}$ Sobolev space that forces $C^{0}$ continuity of solutions and hence square-integrability of the derivatives. On the other hand, DG uses a more relaxed Hilbert $L^{2}=H^{0}$ space, in which square-integrability of the derivatives is not a necessity. In other words, basis functions and solution in the $L^{2}$ space can have jumps across element interfaces; DG uses a local element wise storage scheme that has extra degrees of freedoms to allow for these jumps. Therefore, to obtain a continuous CG solution from within the DG framework, we may apply constraints on duplicate degrees of freedom. Borrowing ideas from the finite element literature $[10,34,42,18]$, we show how equality constraints can be applied to convert a DG formulation into a CG formulation using three different approaches: the masterslave, penalty and Lagrange multiplier methods. We then present a simplified version of the method for the case of a diagonal mass matrix and implement it in NUMA. The implementations are validated with several examples that are representative of typical atmospheric dynamics. Cloud resolving capabilities are tested with a rising thermal bubble problem [14], mesoscale modeling capabilities with a density current problem $[37,13]$ and a global scale capability is tested with an acoustic wave propagation problem run on the entire planet [38].

The remainder of this paper is organized as follows. In Sec. 2, we describe the non-hydrostatic Euler equations used in NUMA. For better stability of the solution, the equations are formulated for a perturbed state about the hydrostatic equilibrium. In Sec. 3, we describe the formulation of the CG and DG spatial discretization methods. Section 4 contains the core of this paper, the unification of CG and DG in NUMA. The implementation of unified CG/DG is described in Sec. 5 and validated with several test cases in Sec. 6 .

\section{Governing equations}

The dynamics of non-hydrostatic atmospheric processes are governed by the compressible Euler equations. Among the five equation sets discussed in [16], NUMA uses three: two conservative sets (Set2C and Set3C) used with both CG and DG discretizations, and a non-conservative equation set used exclusively with CG (Set2NC). In the following, we give an outline of equation Set2C. 
The prognostic variables for equation $\operatorname{Set} 2 \mathrm{C}$ are $(\rho, \mathbf{U}, \Theta)^{\top}$, where $\rho$ is density, $\mathbf{U}=\rho \mathbf{u}, \Theta=\rho \theta$, where $\theta$ is potential temperature and $\mathbf{u}=(u, v, w)^{\top}$ are the velocity components, and the superscript $T$ denotes the transpose operator. Equation Set2C is written as follows

$$
\begin{aligned}
& \frac{\partial \rho}{\partial t}+\nabla \cdot \mathbf{U}=0 \\
& \frac{\partial \mathbf{U}}{\partial t}+\nabla \cdot\left(\frac{\mathbf{U} \otimes \mathbf{U}}{\rho}+P \mathbf{I}_{3}\right)=-\rho g \hat{\mathbf{k}} \\
& \frac{\partial \Theta}{\partial t}+\nabla \cdot\left(\frac{\Theta \mathbf{U}}{\rho}\right)=0
\end{aligned}
$$

where the operator $\otimes$ denotes the tensor (outer) product. The pressure in the momentum equation is obtained from the equation of state that is required to close the system. ${ }^{1}$ The pressure is defined as

$$
P=P_{0}\left(\frac{R \Theta}{P_{0}}\right)^{\gamma}
$$

where $R=c_{p}-c_{v}$ and $\gamma=\frac{c_{p}}{c_{v}}$ for given values for specific heat of pressure and volume of $c_{p}$ and $c_{v}$, respectively.

For better numerical stability, the density, pressure and potential temperature variables are split into background and perturbation components (see, e.g. [13] for details). The time-invariant background components are often obtained by assuming hydrostatic equilibrium, although any other state can be used. Here, we use a hydrostatically balanced background field since the atmospheric is essentially in hydrostatic balance with small perturbations that make it non-hydrostatic. We define the splitting of the background and perturbation variables as follows

$$
\begin{array}{r}
\rho(\mathbf{x}, t)=\bar{\rho}(z)+\rho^{\prime}(\mathbf{x}, t) \\
\Theta(\mathbf{x}, t)=\bar{\Theta}(z)+\Theta^{\prime}(\mathbf{x}, t) \\
P(\mathbf{x}, t)=\bar{P}(z)+P^{\prime}(\mathbf{x}, t)
\end{array}
$$

where $(\mathbf{x}, \mathbf{t})$ are the space-time coordinates ${ }^{2}$. Using this decomposition of the variables, the equation set now takes the following form

$$
\begin{aligned}
& \frac{\partial \rho^{\prime}}{\partial t}+\nabla \cdot \mathbf{U}=0 \\
& \frac{\partial \mathbf{U}}{\partial t}+\nabla \cdot\left(\frac{\mathbf{U} \otimes \mathbf{U}}{\rho}+P^{\prime} \mathbf{I}_{3}\right)=-\rho^{\prime} g \hat{\mathbf{k}} \\
& \frac{\partial \Theta^{\prime}}{\partial t}+\nabla \cdot\left(\frac{\Theta \mathbf{U}}{\rho}\right)=0 .
\end{aligned}
$$

\footnotetext{
${ }^{1}$ Note that, to simplify the exposition, we have omitted the Coriolis terms.

${ }^{2}$ For spherical domains, the coordinate $z$ is replaced by the radial coodinate.
} 
In compact vector notation form, it becomes

$$
\frac{\partial \mathbf{q}}{\partial t}+\nabla \cdot \mathbf{F}=S(\mathbf{q})
$$

where $\mathbf{q}=\left(\rho^{\prime}, \mathbf{U}, \Theta^{\prime}\right)^{\top}$ is the solution vector, $\mathbf{F}=\left(\mathbf{U}, \frac{\mathbf{U} \otimes \mathbf{U}}{\rho}+P^{\prime} \mathbf{I}_{3}, \frac{\Theta \mathbf{U}}{\rho}\right)^{\top}$ is the flux, and $S(\mathbf{q})=\left(0,-\rho^{\prime} g \hat{\mathbf{k}}, 0\right)^{\top}$ is the source vector.

\section{Spatial discretization}

Let us now describe the approach for constructing approximations to the spatial derivatives. We decompose the domain $\Omega \subset \mathbb{R}^{3}$ into $N_{e}$ disjoint hexahedra elements

$$
\Omega=\bigcup_{e=1}^{N_{e}} \Omega_{e} .
$$

Within each element $\Omega_{e}$, basis functions $\psi_{j}(\mathbf{x})$ area defined to form a finitedimensional approximation $\mathbf{q}_{N}$ of $\mathbf{q}(\mathbf{x}, t)$ by the expansion

$$
\mathbf{q}_{N}{ }^{(e)}(\mathbf{x}, t)=\sum_{j=1}^{M} \psi_{j}(\mathbf{x}) \mathbf{q}_{j}^{(e)}(t)
$$

where $M$ is the number of nodes in an element and the superscript $(e)$ denotes an element-based entity. Let us now use this basis function expansion to construct spatial discretization strategies for both CG and DG methods.

\subsection{Continuous Galerkin method}

Starting from the differential form of the Euler equations in vector notation as given in Eq. (4), and then expanding with basis functions, multiplying by a test function $\psi_{i}$, and integrating yields the element-wise formulation

$$
\int_{\Omega_{e}} \psi_{i} \frac{\partial \mathbf{q}_{N}}{\partial t} d \Omega_{e}+\int_{\Omega_{e}} \psi_{i} \nabla \cdot \mathbf{F} d \Omega_{e}=\int_{\Omega_{e}} \psi_{i} S\left(\mathbf{q}_{N}\right) d \Omega_{e} .
$$

Integrating the second term by parts $\left(\psi_{i} \nabla \cdot \mathbf{F}=\nabla \cdot\left(\psi_{i} \mathbf{F}\right)-\nabla \psi_{i} \cdot \mathbf{F}\right)$ yields

$$
\int_{\Omega_{e}} \psi_{i} \frac{\partial \mathbf{q}_{N}}{\partial t} d \Omega_{e}+\int_{\Gamma_{e}} \psi_{i} \hat{\mathbf{n}} \cdot \mathbf{F} d \Gamma_{e}-\int_{\Omega_{e}} \nabla \psi_{i} \cdot \mathbf{F} d \Omega_{e}=\int_{\Omega_{e}} \psi_{i} S\left(\mathbf{q}_{N}\right) d \Omega_{e} .
$$

The second term needs to be evaluated only at physical boundaries because the fluxes to the left and right of element interfaces are always equal at interior boundaries, i.e. $\mathbf{F}^{+}=\mathbf{F}^{-}$. Eqs. (5) and (6) are the strong and weak continuous Galerkin (CG) formulations, respectively, with the finite dimensional space defined as a subset of the Sobolev space 


$$
\nu_{N}^{C G}=\left\{\psi \in H^{1}\left(\Omega_{e}\right) \mid \psi \in P_{N}(I), e=1, \ldots, N_{e}\right\}
$$

where $P_{N}$ defines the set of all $N$ th degree polynomials. Automatically, $\nu_{N}^{C G} \in$ $C^{0}\left(\Omega_{e}\right)$, thus CG solutions satisfy $C^{0}$-continuity.

\subsection{Discontinuous Galerkin method}

For DG, the finite dimensional space is defined as a subset of the Hilbert space that allows for discontinuities of solutions

$$
\nu_{N}^{D G}=\left\{\psi \in L^{2}\left(\Omega_{e}\right) \mid \psi \in P_{N}(I), e=1, \ldots, N_{e}\right\} .
$$

Therefore $\mathbf{F}^{+}$and $\mathbf{F}^{-}$are not equal anymore. A numerical flux $\mathbf{F}^{*}$ is defined as an approximate solution to a Riemann problem to be used in the weak form DG

$$
\int_{\Omega_{e}} \psi_{i} \frac{\partial \mathbf{q}_{N}}{\partial t} d \Omega_{e}+\int_{\Gamma_{e}} \psi_{i} \hat{\mathbf{n}} \cdot \mathbf{F}^{*} d \Gamma_{e}-\int_{\Omega_{e}} \nabla \psi_{i} \cdot \mathbf{F} d \Omega_{e}=\int_{\Omega_{e}} \psi_{i} S\left(\mathbf{q}_{N}\right) d \Omega_{e}
$$

where the Rusanov flux, suitable for hyperbolic equations, is defined as

$$
\mathbf{F}^{*}=\frac{1}{2}\left[\mathbf{F}^{+}+\mathbf{F}^{-}-\hat{\mathbf{n}} c_{\max }\left(\mathbf{q}^{+}-\mathbf{q}^{-}\right)\right]
$$

with $c_{\max }=|\hat{\mathbf{n}} \cdot \mathbf{u}|+a$ is the maximum wave speed, and $a$ is the speed of sound. If $C^{0}$-continuity is enforced on the weak form $\mathrm{DG}$ in Eq. (7), i.e. $\mathbf{F}=\mathbf{F}^{*}$, it reduces to the weak form CG in Eq. (6).

A strong form DG that resembles Eq. (5) more, can be obtained by applying a second integration by parts on the flux integral to remove the smoothness constraint on the test function $\psi_{i}$ as follows

$$
\int_{\Omega_{e}} \psi_{i} \frac{\partial \mathbf{q}_{N}}{\partial t} d \Omega_{e}+\int_{\Gamma_{e}} \psi_{i} \hat{\mathbf{n}} \cdot\left(\mathbf{F}^{*}-\mathbf{F}\right)\left(\mathbf{q}_{N}\right) d \Gamma_{e}+\int_{\Omega_{e}} \psi_{i} \nabla \cdot \mathbf{F}\left(\mathbf{q}_{N}\right) d \Omega_{e}=\int_{\Omega_{e}} \psi_{i} S\left(\mathbf{q}_{N}\right) d \Omega_{e}
$$

Again, if $C^{0}$-continuity is enforced on the strong form DG formulation, i.e $\mathbf{F}=$ $\mathbf{F}^{*}$ at interior edges, it simplifies to the strong form CG formulation in Eq. (5). ${ }^{3}$

\subsection{Boundary conditions}

All the test cases we use to validate our implementations are what are commonly known as flow in a box type in which no mass or energy leaves the computational domain. If, then, we determine that mass or energy is being lost during a simulation, it is indicative of one of the following: the presence of numerical errors for those equation sets that are conservative by construction, and

\footnotetext{
DG.

${ }^{3}$ Using Eq. (8), we can enforce boundary conditions via the flux integrals for both CG and
} 
the deviation of the numerical solution from the analytical solution for those equation sets that are non-conservative [15]. The equation set we use for this study is Set2C which is locally conservative and thus falls under the first group. The required boundary condition is a no-flux (NFBC) type in which the flow remains constrained inside the domain and the surface of the boundary offers no viscous resistance to the fluid flow. NFBCs force the normal component of velocity to go to zero at all physical boundaries $\Gamma$ as such

$$
\hat{\mathbf{n}} \cdot \mathbf{u}=0
$$

where $\hat{\mathbf{n}}=\left(n_{x}, n_{y}, n_{z}\right)^{\top}$ is the outward pointing unit normal vector on $\Gamma$. The NFBCs are applied to the standard CG formulation using an orthogonal projector matrix computed as

$$
P=\mathbf{I}-\hat{\mathbf{n}} \otimes \hat{\mathbf{n}} .
$$

The NFBCs may be applied in a strong sense directly on the velocity vector in the interior of the domain. For DG, we impose NFBCs on velocity using ghost cells on which the velocity is set as a reflection of the one inside of the wall, i.e.

$$
P=\mathbf{I}-2 \hat{\mathbf{n}} \otimes \hat{\mathbf{n}}
$$

known as a reflector in linear algebra. The above two ways of imposing NFBCs should give similar results; however, the DG way of imposing NFBCs is better at corner points for the following reason. If we compute a numerical flux at corner nodes shared by two faces, each get its own numerical flux and that flux is set to zero to impose the NFBCs. However, using the CG way, the contribution from the two faces will be the sum total of the contributions from the two faces. Hence, if an NFBC is imposed at a corner node of a rectangular element, the result will be a flow directed at $45^{0}$ into or out of the domain unless a fix is added to avoid this situation. Using a unified CG/DG formulation simplifies the application of NFBCs for the CG method because they are handled in an identical manner to DG.

\subsection{Stabilization}

Godunov's theorem states that numerical schemes for solving PDEs that have the property of not generating new extrema, so called monotone schemes, are at most first order accurate. This result had a major impact on the development of high order schemes for Computational Fluid Dynamics (CFD). High order CG and DG methods suffer from this limitation especially when used for solving convection dominated flows. According to [20], the method often used in operational NWP models to stabilize CG and DG is to add second or higherorder artificial diffusion (AD) to the right hand side of Eq. (3). For example, one would add the term (see, e.g., [14])

$$
A D=\left[\begin{array}{c}
0 \\
\nabla \cdot(\rho \nu \nabla \mathbf{u}) \\
\nabla \cdot\left(\rho \nu \nabla \theta^{\prime}\right)
\end{array}\right]
$$


where $\nu$ is an artificial kinematic viscosity which is assumed to be constant for this work. Adaptive methods exist in which $\nu$ is selected, for instance, based on the Peclet number. We do not add artificial diffusion to the mass continuity equation to ensure mass conservation in all cases. Plain DG without AD can be more stable than CG because DG gets implicit dissipation from its use of an upwind-biased numerical flux.

Spurious oscillations in high order methods are a result of aliasing errors introduced when the grid is coarse and the numerical scheme interprets high frequency waves as if they were low frequency waves. A second method of stabilization, which is used for stabilizing the acoustic wave test case in Section 6.3 , is the use of filters, which may be more scale sensitive $[11,4,5]$ than second order diffusion. Filters transform the solution into the frequency domain, curtail troublesome high frequency noise using a low pass filter, and then transform the result back to the physical space.

\section{Unified CG and DG}

Now that we have described the basic building-blocks for both the CG and DG methods, let us now describe how to construct a unified formulation of these two methods. The element wise matrices for both CG and DG are assembled to form global matrices through an operation commonly known as global assembly or direct stiffness summation (DSS). CG is often implemented through a global grid point storage scheme, where elements share the nodes ${ }^{4}$ at faces thereby ensuring $C^{0}$-continuity inherently. Thus, the DSS operation for CG accumulates values at the shared nodes, while that for DG simply puts the local element matrices in their proper location in the global matrix. DG uses a local element wise storage scheme because discontinuities (jumps) at element interfaces are allowed. The standard implementation of CG and DG often follow these two different approaches of storing data; however, CG can be recast to use local element wise storage like DG. So how do we achieve this goal?

We wish to convert the DG formulation into a CG formulation so that a DG code can produce a $C^{0}$ continuous solution in which fluxes disappear at all element interfaces that are not physical boundaries. This is not the same as enforcing $C^{0}$ continuity of a final DG solution by taking simple or weighted averages for the sake of better visualization of results. The $C^{0}$ continuous result we get using this simplified method is not the same as the one we get using the CG formulation, because the constraint needs to be enforced for every evaluation of the vectors. Nonetheless, it points us to a method in which the averaging on the mass matrix and right hand side vector are done after every computation of the vectors instead of just once at the end of simulation. The simple averaging method will work in cases where the mass matrix is diagonal, but a more rigorous approach is required when implicit terms make the matrix non-diagonal. As

\footnotetext{
${ }^{4}$ In this work, we rely on the Legendre-Gauss-Lobatto (LGL) points as the nodes because it allows us to use the same set of points for both CG and DG.
} 
mentioned in the previous section, the strong form DG formulation reduces to the CG formulation if $C^{0}$ continuity is enforced on the fluxes, i.e. $\mathbf{F}=\mathbf{F}^{*}$.

DG has more degrees of freedom than CG because the finite dimensional space for CG, $H^{1}$, is a subset of that of DG's, $L^{2}$. The key idea here is to apply a constraint on DG to reduce the finite dimensional space to $H^{1}$ so that we get a $C^{0}$ continuous solution. We emphasize here that this is a constraint application or imposition process similar to that done at physical boundaries to apply constraints, also known as boundary conditions. Therefore, the only difference here is that the constraint we need is applied on all element interfaces instead of just physical boundaries. In the following, we discuss different methods of applying this constraint.

Let us first define this constraint. For a conforming grid where corresponding LGL nodes at faces reside at the same location, the constraint can be thought of as an equality enforcing constraint on the left and right nodes, i.e $\mathbf{q}^{\mathbf{L}}=\mathbf{q}^{\mathbf{R}}$. For a non-conforming grid, with hanging nodes, the constraint is a general linear equation. For LGL nodes located at corners, and edges of elements, there are more than two elements sharing a node so we will have multiple constraints per node. The constraint on a corner node of a $2 \mathrm{D}$ element are shared by four elements NE, SE, SW and NW of the node as follows

$$
\begin{aligned}
& q^{N E}=q^{S E} \\
& q^{N E}=q^{S W} \\
& q^{N E}=q^{N W} .
\end{aligned}
$$

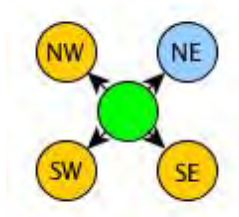

Figure 2: DG to CG node mapping

In general, if the spatial dimension of the problem is $\mathrm{d}$, we will require a maximum of $2^{d}-1$ independent constraints per node. In the finite element literature, these constraints are known as multi-freedom equality constraints (MFCs) $[10,34,42]$. They are also homogenous constraints. Using a transformation matrix $\mathbf{T}$, the constraint can be represented in the compact notation

$$
\mathbf{q}_{\mathbf{d g}}=\mathbf{T q}_{\mathbf{c g}}
$$

where $\mathbf{q}_{\mathbf{d g}}$ is the solution vector ( e.g., pressure, velocity, ...) of DG, and $\mathbf{q}_{\mathbf{c g}}$ is the counterpart for CG with reduced degrees of freedom. ${ }^{5}$ The spaces of the

\footnotetext{
${ }^{5}$ The matrix $\mathbf{T}$ described here is the same scatter matrix defined as $\mathbf{Q}$ in [25].
} 
vectors and matrix are $\mathbf{q}_{\mathbf{d g}} \in \mathbb{R}^{N_{d g}}, \mathbf{q}_{\mathbf{c g}} \in \mathbb{R}^{N_{c g}}$, and $\mathbf{T} \in \mathbb{R}^{N_{d g} \times N_{c g}}$ where $N_{d g}$ and $N_{c g}$ are the dimension of the solution vectors for DG and CG respectively.

The discretized system of linear equations for DG and CG can be represented as

$$
\begin{aligned}
\mathbf{K}_{\mathrm{dg}} \mathbf{q}_{\mathrm{dg}} & =\mathbf{R}_{\mathrm{dg}} \\
\mathbf{K}_{\mathrm{cg}} \mathbf{q}_{\mathrm{cg}} & =\mathbf{R}_{\mathrm{cg}}
\end{aligned}
$$

where $\mathbf{R}_{\mathbf{d g}}, \mathbf{R}_{\mathbf{c g}}$ are the right hand side vectors, $\mathbf{K}_{\mathbf{d g}}, \mathbf{K}_{\mathbf{c g}}$ are the stiffness matrices. The stiffness matrix will be the mass matrix when all terms in the PDE except the temporal derivative are discretized explicitly, i.e. they are all on the right hand side. We seek to condense the DG set of equations to CG by applying an MFC constraint

$$
\mathbf{K}_{\mathbf{d g}} \mathbf{q}_{\mathbf{d g}}=\mathbf{R}_{\mathbf{d g}} \stackrel{M F C s}{\longrightarrow} \mathbf{K}_{\mathbf{c g}} \mathbf{q}_{\mathbf{c g}}=\mathbf{R}_{\mathbf{c g}} .
$$

\subsection{Methods of applying constraints}

There are different ways of enforcing equality constraints on a DG formulation to convert it into a CG formulation. We borrow some constraint imposition techniques from the finite element literature $[42,10]$ and discuss how they can be applied to a DG formulation. The methods vary in complexity of implementation and accuracy of enforcing the constraint. Simple methods such as the penalty method can be robust but are not accurate, in the sense that the constraint is not satisfied exactly [10]. In the following, three commonly used techniques are described using a general stiffness matrix that could be the result of a DG discretization. After that, we describe the special case for diagonal matrices, which is the method used in NUMA. A more general approach for non-diagonal matrices will be described in a separate paper.

\subsubsection{Master-slave method}

In this method, we choose one of the duplicate nodes in the constraint equation to be a master and eliminate all others by applying equality constraints. For example, in Eq. (10), the north-east node is the master against which the equality constraint for all other nodes is formulated. Thus, the transformation for one corner node, shown in Fig. 2, shared by four elements can be written as

$$
\begin{aligned}
{\left[\begin{array}{c}
\mathbf{q}^{\mathbf{N E}} \\
\mathbf{q}^{\mathbf{S E}} \\
\mathbf{q}^{\mathbf{S W}} \\
\mathbf{q}^{\mathbf{N W}}
\end{array}\right] } & =\left[\begin{array}{l}
1 \\
1 \\
1 \\
1
\end{array}\right]\left[\mathbf{q}^{\mathbf{N E}}\right] \\
\mathbf{q}_{\mathbf{d g}} & =\mathbf{T} \mathbf{q}_{\mathbf{c g}}
\end{aligned}
$$

where $N_{d g}=4$ and $N_{c g}=1$ for this particular case. Though the transformation matrix $\mathbf{T}$ looks like a vector for this degenerate example, it is actually a rectangular matrix living in the space of $\mathbb{R}^{N_{d g} \times N_{c g}}$ in the general case. See App. 
A for an example demonstrating the application of the master-slave elimination method.

Once the transformation matrix is built, we apply the constraint as follows. Multiplying both sides of the constraint equation Eq. (11) by $\mathbf{T}^{\top} \mathbf{K}_{\mathrm{dg}}$ yields

$$
\left(\mathbf{T}^{\top} \mathbf{K}_{\mathrm{dg}}\right) \mathbf{q}_{\mathrm{dg}}=\left(\mathbf{T}^{\top} \mathbf{K}_{\mathrm{dg}}\right) \mathbf{T} \mathbf{q}_{\mathbf{c g}} .
$$

Substituting Eq. (12) to the left hand side yields

$$
\left(\mathbf{T}^{\top} \mathbf{K}_{\mathrm{dg}} \mathbf{T}\right) \mathbf{q}_{\mathrm{cg}}=\mathbf{T}^{\top} \mathbf{R}_{\mathrm{dg}} .
$$

Therefore, we can write

$$
\begin{aligned}
& \mathbf{K}_{\text {cg }}=\mathbf{T}^{\top} \mathbf{K}_{\mathrm{dg}} \mathbf{T} \\
& \mathbf{R}_{\mathbf{c g}}=\mathbf{T}^{\top} \mathbf{R}_{\mathrm{dg}} .
\end{aligned}
$$

The transformation is now complete with the above relation for the stiffness matrix and right hand side of a DG and CG formulation. The transformation for the stiffness matrix is known as a congruent transformation in mathematics. Let us now look closely at what happens inside the DG stiffness matrix $\mathbf{K}_{\mathbf{d g}}$ when we apply the transformation. For this purpose, we divide the LGL nodes into three sets: internal nodes in elements represented by the index $i$, master LGL nodes by $m$ and slave LGL nodes by $s$. The stiffness matrix can be rearranged where internal degrees of freedom are first followed by master and then slave degrees of freedom. This rearrangement causes difficulty in implementation, but we are not concerned about it for now and so we write

$$
\left[\begin{array}{ccc}
\mathbf{K}_{\mathrm{ii}} & \mathbf{K}_{\mathrm{im}} & \mathbf{K}_{\mathrm{is}} \\
\mathbf{K}_{\mathrm{mi}} & \mathbf{K}_{\mathrm{mm}} & \mathbf{K}_{\mathrm{ms}} \\
\mathbf{K}_{\mathrm{si}} & \mathbf{K}_{\mathrm{sm}} & \mathbf{K}_{\mathrm{ss}}
\end{array}\right]\left[\begin{array}{c}
\mathbf{q}_{\mathbf{i}} \\
\mathbf{q}_{\mathbf{m}} \\
\mathbf{q}_{\mathrm{s}}
\end{array}\right]=\left[\begin{array}{c}
\mathbf{R}_{\mathbf{i}} \\
\mathbf{R}_{\mathbf{m}} \\
\mathbf{R}_{\mathbf{s}}
\end{array}\right] .
$$

Let us first define the transformation matrix directly on the master and slave nodes, i.e. excluding the internal nodes which are mapped to themselves anyway

$$
\mathbf{q}_{\mathrm{s}}=\mathbf{T} \mathbf{q}_{\mathrm{m}}
$$

Substituting Eq. (11) and multiplying the third row by $\mathbf{T}^{\top}$ yields a condensed matrix in which the slave degrees of freedom are eliminated

$$
\left[\begin{array}{cc}
\mathbf{K}_{\mathbf{i i}} & \mathbf{K}_{\mathbf{i m}}+\mathbf{K}_{\mathbf{i s}} \mathbf{T} \\
\mathbf{K}_{\mathbf{m i}}+\mathbf{T}^{\top} \mathbf{K}_{\mathbf{s i}} & \mathbf{K}_{\mathbf{m m}}+\mathbf{K}_{\mathbf{m s}} \mathbf{T}+\mathbf{T}^{\top} \mathbf{K}_{\mathbf{s m}}+\mathbf{T}^{\top} \mathbf{K}_{\mathbf{s s}} \mathbf{T}
\end{array}\right]\left[\begin{array}{c}
\mathbf{q}_{\mathbf{i}} \\
\mathbf{q}_{\mathbf{m}}
\end{array}\right]=\left[\begin{array}{c}
\mathbf{R}_{\mathbf{i}} \\
\mathbf{R}_{\mathbf{m}}+\mathbf{T}^{\top} \mathbf{R}_{\mathbf{s}}
\end{array}\right] .
$$

\subsubsection{Penalty method}

In the finite element literature, the physical interpretation of this method is to connect constrained nodes with a bar of very high stiffness (penalty element) [42]. Penalties were first introduced to finite elements by Lions [26] as a means of imposing Dirichlet boundary conditions weakly. Since then penalties have 
found use in DG methods for elliptic equations to enforce continuity of solution and its derivative, so called interior penalty methods [35]. Using this method with a finite penalty weight $w$, the constraint will not be satisfied exactly but only approximately. In the same manner as the interior penalty methods, we can apply penalties here to enforce $C^{0}$ continuity on a DG formulation. To apply the penalty method on the re-arranged DG stiffness matrix in Eq. (17), we add penalty weights as follows

$$
\left[\begin{array}{ccc}
\mathbf{K}_{\mathbf{i i}} & \mathbf{K}_{\mathbf{i m}} & \mathbf{K}_{\mathbf{i s}} \\
\mathbf{K}_{\mathbf{m i}} & \mathbf{K}_{\mathbf{m m}}+w \mathbf{I}_{\mathbf{m m}} & \mathbf{K}_{\mathbf{m s}}-w \mathbf{I}_{\mathbf{m s}} \\
\mathbf{K}_{\mathbf{s i}} & \mathbf{K}_{\mathbf{s m}}-w \mathbf{I}_{\mathbf{s m}} & \mathbf{K}_{\mathbf{s s}}+w \mathbf{I}_{\mathbf{s s}}
\end{array}\right]\left[\begin{array}{c}
\mathbf{q}_{\mathbf{i}} \\
\mathbf{q}_{\mathbf{m}} \\
\mathbf{q}_{\mathbf{s}}
\end{array}\right]=\left[\begin{array}{c}
\mathbf{R}_{\mathbf{i}} \\
\mathbf{R}_{\mathbf{m}} \\
\mathbf{R}_{\mathbf{s}}
\end{array}\right] .
$$

A large penalty term within computer overflow limits will dominate all other terms in the linear equation which do not have penalty weights themselves, thereby, satisfying the constraint approximately. Then it seems the degree by which the constraint is satisfied increases with the penalty weight. However, the stiffness matrix becomes ill-conditioned with extremely large penalty weight. The second and third rows of the stiffness matrix in Eq. (19) will become linearly dependent, one being the negative of the other, when $w \gg 1$. Therefore the selection of the penalty weight becomes a tradeoff between two competing goals of reducing the constraint violation error and maintaining a low condition number of the stiffness matrix for the solver.

Rewriting the homogeneous constraints in Eq. (11) using a constraint matrix C defined in the space of $\mathbb{R}^{m \times N_{d g}}$ for $m$ constraints, we get

$$
\mathbf{C q}_{d g}=\mathbf{0}
$$

which allows us to write the stiffness matrix for the penalty method as

$$
\mathbf{K}_{p}=\mathbf{K}_{d g}+\mathbf{C}^{\top} \mathbf{W C}
$$

where $\mathbf{W}=w \mathbf{I} \in \mathbb{R}^{m \times m}$.

\subsubsection{Lagrange multiplier method}

This method, first used for finite elements in [18], is conceptually similar to the penalty method but it has the ability to satisfy the constraints exactly. As discussed before, the penalty method will not yield a solution when using an infinite penalty weight. The idea behind this method is to calculate the forcing required to satisfy the constraint exactly, henceforth known as Lagrange multipliers $\lambda$, by augmenting the stiffness matrix with the constraint equations and solving for both $q$ and $\lambda$. Given the original discretized system of equations

$$
\mathbf{K}_{d g} \mathbf{q}_{d g}=\mathbf{R}_{d g}
$$

we add the the constraint as follows

$$
\mathbf{K}_{d g} \mathbf{q}_{d g}+\mathbf{C}^{\top} \lambda=\mathbf{R}_{d g} .
$$

Then, the stiffness matrix is bordered with $\mathbf{C}$ and $\mathbf{C}^{\top}$ as follows 


$$
\left[\begin{array}{cc}
\mathbf{K}_{\mathbf{d g}} & \mathbf{C}^{\top} \\
\mathbf{C} & \mathbf{0}
\end{array}\right]\left[\begin{array}{c}
\mathbf{q}_{\mathbf{d g}} \\
\lambda
\end{array}\right]=\left[\begin{array}{c}
\mathbf{R}_{\mathbf{d g}} \\
\mathbf{0}
\end{array}\right]
$$

Even though this method satisfies the constraints exactly, it requires additional unknowns (the Lagrange multipliers). Moreover, the augmented matrix becomes indefinite which is problematic for linear equation solvers that depend on positive definiteness.

The mortar finite element method of Bernardi et al. [3] uses Lagrange multipliers to impose coupling between two domains that use different discretization methods on non-overlapping sub-domains, for instance spectral elements on one part and finite difference on the other. The methods has applications in problems such as fluid-structure interactions and multi-phase flows, and can handle different types of non-conformities between sub-domains.

\subsection{Relation to static condensation}

The model reduction process from DG to CG looks similar to another model reduction method known as static condensation [34] in the FEM literature. However, static condensation (SC) does not apply a constraint to enforce $C^{0}$ continuity. With that in mind, static condensation, when applied to a DG discretized set of equations in cases where it is applicable, should yield a discontinuous DG solution similar to that which one would get without its application. To see the relation of this method to the master-slave method, let us apply it to the DG discretized set of equations

$$
\mathbf{K}_{\mathrm{dg}} \mathbf{q}_{\mathbf{d g}}=\mathbf{R}_{\mathrm{dg}} \stackrel{S C s}{\longrightarrow} \hat{\mathbf{K}}_{\mathrm{dg}} \hat{\mathbf{q}}_{\mathbf{d g}}=\hat{\mathbf{R}}_{\mathrm{dg}} .
$$

Selecting master and slave nodes, we have the DG formulation before transformation

$$
\left[\begin{array}{cc}
\mathbf{K}_{\mathrm{mm}} & \mathbf{K}_{\mathrm{ms}} \\
\mathbf{K}_{\mathrm{sm}} & \mathbf{K}_{\mathrm{ss}}
\end{array}\right]\left[\begin{array}{c}
\mathbf{q}_{\mathrm{m}} \\
\mathbf{q}_{\mathrm{s}}
\end{array}\right]=\left[\begin{array}{c}
\mathbf{R}_{\mathbf{m}} \\
\mathbf{R}_{\mathrm{s}}
\end{array}\right] .
$$

The static condensation process yields a stiffness matrix that is the Schur complement of $\mathbf{K}_{\mathrm{ss}}$

$$
\begin{aligned}
& \hat{\mathbf{K}}_{\mathrm{dg}}=\mathbf{K}_{\mathrm{mm}}-\mathbf{K}_{\mathrm{ms}} \mathbf{K}_{\mathrm{ss}}{ }^{-1} \mathbf{K}_{\mathrm{sm}} \\
& \hat{\mathbf{R}}_{\mathrm{dg}}=\mathbf{R}_{\mathrm{m}}-\mathbf{K}_{\mathrm{ms}} \mathbf{K}_{\mathrm{ss}}{ }^{-1} \mathbf{R}_{\mathrm{s}} .
\end{aligned}
$$

The static condensation process can be thought of as a special case of the master-slave method applied with an inhomogeneous constraint calculated from the matrix coefficients themselves. So this is an artificial constraint unlike the external constraints applied in previous sections to force a DG formulation to respect $C^{0}$ continuity. The required constraint is inhomogeneous with a nonzero right hand side value $\mathbf{r}$

$$
\mathbf{q}_{\mathrm{s}}=\mathbf{T} \mathbf{q}_{\mathbf{m}}+\mathbf{r}
$$


where

$$
\mathbf{T}=-\mathbf{K}_{\mathrm{ss}}{ }^{-1} \mathbf{K}_{\mathrm{sm}} \text {, and } \mathbf{r}=\mathbf{K}_{\mathrm{ss}}{ }^{-1} \mathbf{R}_{\mathrm{s}}
$$

\subsection{Relation to Hybridizable Discontinuous Galerkin (HDG)}

Hybridizable Discontinuous Galerkin (HDG) [7, 23, 32, 31, 40] is a method that aims to make DG computationally competitive with CG using the static condensation model reduction technique discussed in the previous section. Hybridization was introduced in [41] for efficient solution of finite element approximations of linear elasticity problems. Both HDG and CG with static condensation follow the same pipeline [31, 40], namely construction of local problems, global formulation and post-processing. The local problem is the procedure to find the solution on an element based on the solution at the boundary assuming fixed values. In the global formulation, the primary unknowns are defined on the boundaries (trace) of the elements, whereas internal degrees of freedom are eliminated from the global system by employing the Schur complement of the assembled system. CG methods have benefited from this model reduction technique for a long time before DG methods started to mimic the procedure with the introduction of HDG in [7]. Once the global solution on the boundaries is obtained by solving the statically condensed global system of equations, the solution in the internal degrees of freedom can be recovered independently in each element. It is demonstrated in [23] that the HDG method can be made to be as efficient as the CG method that uses static condensation at moderate polynomial orders.

\subsection{NUMA's unified $C G / D G$ method}

In element-based Galerkin methods, coupling neighboring elements is achieved by specifying either the solution vector $\mathbf{q}$ in case of CG (strong coupling) or the numerical flux $\mathbf{F}^{*}$ in case of DG (weak coupling). NUMA uses a diagonal lumped mass matrix approach for efficiency reasons; inversion of a diagonal mass matrix is much simpler. This is achieved through the use of inexact integration in which interpolation and quadrature points are collocated at the LegendreGauss-Lobatto (LGL) points. Moreover, the required coupling for CG and DG are conducted explicitly, in which their contribution is moved to the right hand side vector. This makes converting a DG formulation to CG formulation in NUMA trivial. Starting from the result of the master-slave method in Eq. (18) and removing all off-diagonal terms, we get

$$
\left[\begin{array}{cc}
\mathbf{K}_{\mathbf{i i}} & \mathbf{0} \\
\mathbf{0} & \mathbf{K}_{\mathbf{m m}}+\mathbf{T}^{\top} \mathbf{K}_{\mathbf{s s}} \mathbf{T}
\end{array}\right]\left[\begin{array}{c}
\mathbf{q}_{\mathbf{i}} \\
\mathbf{q}_{\mathbf{m}}
\end{array}\right]=\left[\begin{array}{c}
\mathbf{R}_{\mathbf{i}} \\
\mathbf{R}_{\mathbf{m}}+\mathbf{T}^{\top} \mathbf{R}_{\mathbf{s}}
\end{array}\right] .
$$

For a conforming grid, where one master node is mapped to exactly one slave node, the transformation matrix $\mathrm{T}$ is the identity matrix, which when substituted to the above equation yields

$$
\left[\begin{array}{cc}
\mathbf{K}_{\mathbf{i i}} & \mathbf{0} \\
\mathbf{0} & \mathbf{K}_{\mathbf{m m}}+\mathbf{K}_{\mathrm{ss}}
\end{array}\right]\left[\begin{array}{c}
\mathbf{q}_{\mathrm{i}} \\
\mathbf{q}_{\mathbf{m}}
\end{array}\right]=\left[\begin{array}{c}
\mathbf{R}_{\mathbf{i}} \\
\mathbf{R}_{\mathbf{m}}+\mathbf{R}_{\mathrm{s}}
\end{array}\right] .
$$


The effect of the gather operation is clearly seen with addition of the right hand side vector and stiffness coefficients of the master and slave nodes at boundaries of elements. We should note here that the re-arrangement to internal and boundary degrees of freedom is unnecessary because the operation is simple to apply.

From NUMA's point of view, both the mass matrix and right hand side are stored as vectors, therefore, the operation is the same for both. If we are to remain in the DG framework, after the gather operation is complete, we should send back the accumulated values to the duplicate nodes in the DG framework. We call this operation scatter, and the combined gather-scatter operation is called Direct Stiffness Summation (DSS). ${ }^{6}$ This operation gathers values at local LGL nodes sharing the same global identification number, and then distributes the result back to duplicate LGL nodes so that all will have the same value, which is the sum total. DSS is the coupling mechanism for CG, without which the problem will be ill-posed. DG achieves the same via the definition of the numerical flux $\mathbf{F}^{*}$ at element interfaces; the same numerical flux is used by all elements sharing a face. We note that the classical implementation of CG that uses global nodes, henceforth called CGc ("c" denotes a continuous data storage approach), does the DSS operation naturally. Comparing the number of operations of CGc against CGd (" $d$ " denotes a discontinuous data storage approach - the same storage used in DG) done using DSS from within the DG framework, we can observe that both do the gather operation but the latter requires an additional scatter operation to distribute the sum total values back to slave LGL nodes.

When the mass matrix is non-diagonal or other terms in the governing equation are evaluated implicitly thereby giving rise to a non-diagonal matrix, the DSS operation is extended to gather and scatter off-diagonal terms as well. In this case, the structure of the assembled matrix will not be block-diagonal anymore. An example of applying the DSS operation for an implicit problem is given in Appendix A.

\section{Implementation}

We do not create and store matrices in NUMA but evaluate the action of different finite element operators directly. As is noted in [2], this is more efficient than creating and assembling stiffness matrices. When Krylov subspace methods, such as Conjugate Gradient and Generalized Minimal Residual Method, are used for solving linear systems of equations resulting from discretization of PDEs, one requires to evaluate only the action of a matrix-vector product. A comparison of different schemes to compute the matrix action, in so called matrix-free methods, is discussed in [22].

Prognostic variables in NUMA are stored in a one-dimensional array that is accessed using an index calculated from the element number (e) and location of

${ }^{6}$ In [25] we denote the scatter-gather operation as the matrix operation $Q^{\top} Q$. 
the LGL point $(i, j, k)$. As discussed previously, a classical CG implementation uses a global grid point storage scheme (CGc). To unify CG into the DG framework which uses element wise storage, a local-to-global grid point mapping is required to apply the required DSS operator. For this purpose, an integer matrix intma $(i, j, k, e)$ is pre-calculated at program start up. On the other hand, DG uses a one-to-one mapping where each LGL node in an element has a distinct place in the global vector, hence, a multi-dimensional array $q(i, j, k, e)$ will suffice to implement DG alone. To incorporate CGc into the DG framework, we have to use a one dimensional array $q(I)$, where the index $I$ is either explicitly calculated from $(i, j, k, e)$ for DG or read from the pre-calculated indirect-addressing table intma $(i, j, k, e)$. The standard multi-dimensional array implementation of DG is actually equivalent to the one dimensional array implementation because compilers calculate a single index $I$ to access the multi-dimensional array as if it was a one dimensional array. Given polynomial orders $N_{x}, N_{y}, N_{z}$ in the $x, y, z$ directions and a pre-calculated mapping table intma, the index calculation for CG and DG are shown in Alg. 1 and Alg. 2.
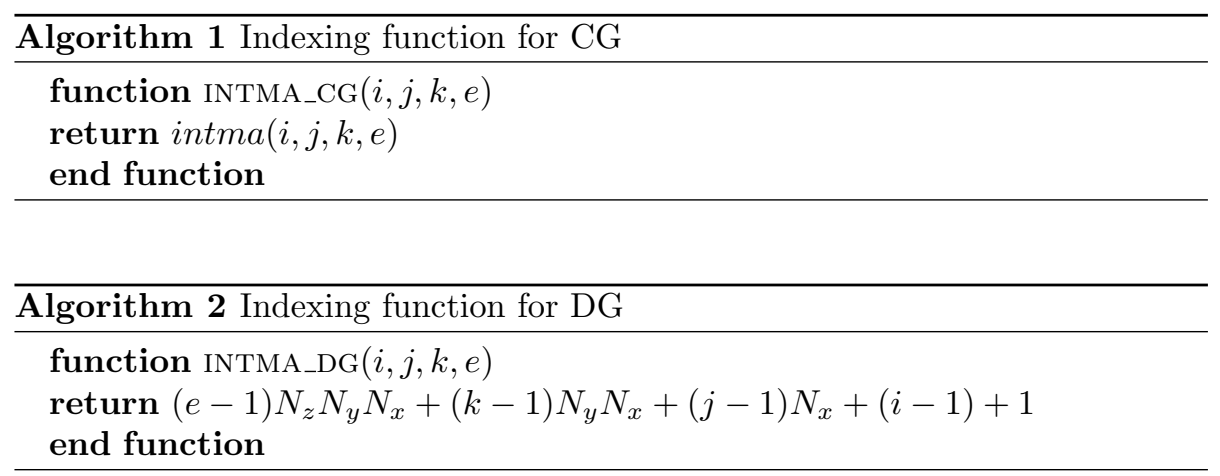

NUMA supports three spatial discretization schemes through appropriate selection of local-to-global grid point mapping functions. The classical CG implementation CGc uses global grid point storage and intma_cg for indirect addressing. The unified CG method that uses local element wise storage, henceforth called CGd, uses intma_dg but then applies the DSS operator, using intma_cg, on the right-hand-side vector and the mass matrix. The CGd method is further classified into two groups based on the way boundary conditions are applied. What we call CGd-unified can use natural (flux) boundary conditions from DG to improve/simplify mass and energy conservation properties compared to the original CGc method. The reason has been explained in Section 3.3. The other method we call CGd-separate applies flux boundary conditions in a similar way as CGc, therefore, it should yield mass and energy conservation properties similar to that of CGc. The DG method uses intma_dg for indexing and calculates inter-element fluxes instead of applying a DSS operator that is required for CG. And finally, we have a hybrid CGDG method in which CG is used in some parts of the domain and DG in others. This is implemented by specifying for each node to use either the strong coupling of CG (DSS operation) or the weak cou- 
pling of DG through fluxes as described in Algorithm 4. For the current work, we use a statically initialized coupling selection algorithm that assigns different regions of the domain to use either CG or DG; however, a dynamic algorithm based on flow characteristics can be used.

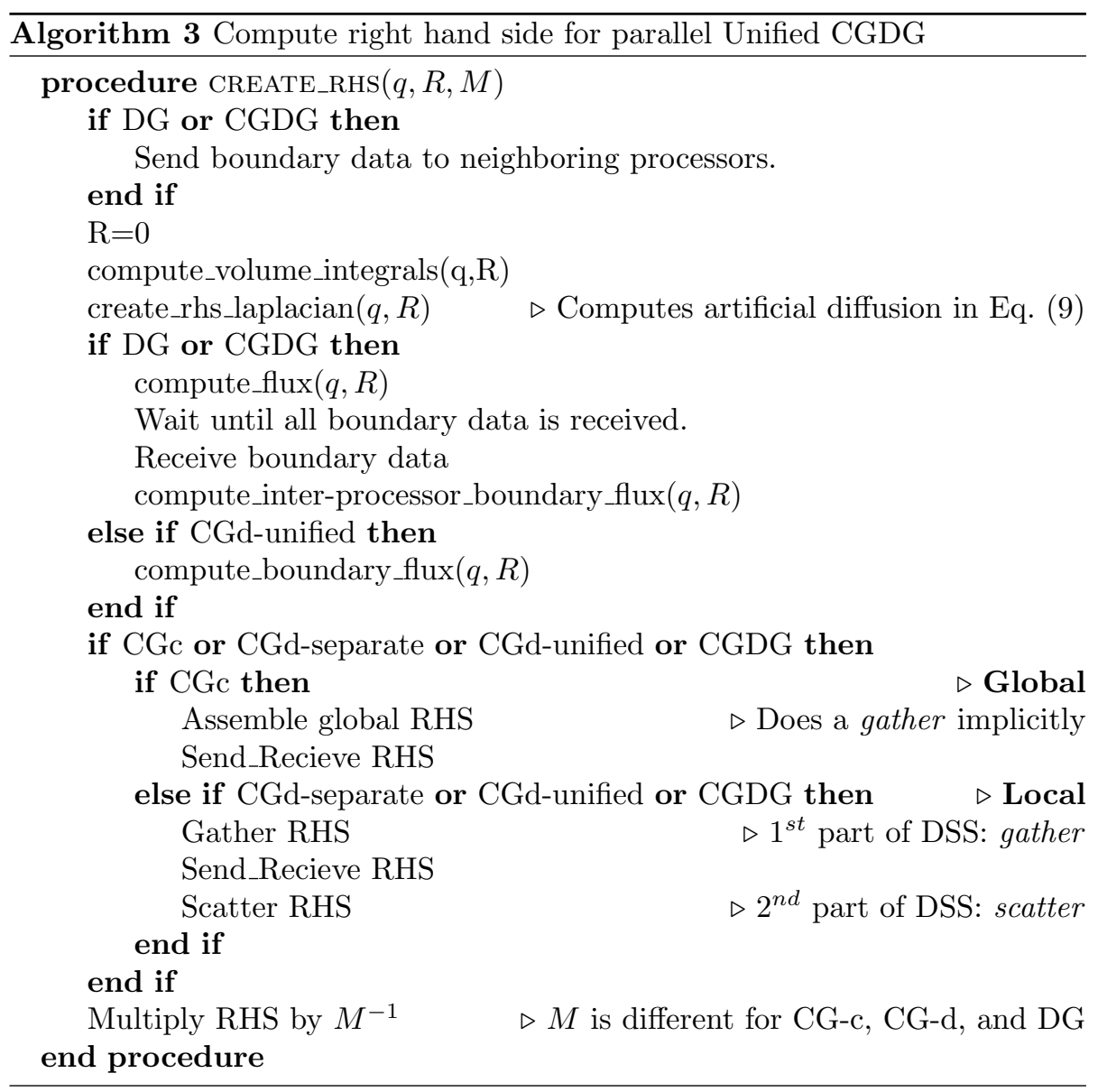

The parallel algorithm for computing the right hand side of a unified CG/DG implementation is shown in Alg. 3. The details of the parallel implementations of CG and DG in NUMA can be found in [21]. DG benefits from a communicationcomputation overlap while computing fluxes. The flux computation subroutines are split into two: inter-processor boundary flux calculation which requires upto-date data from neighboring processors, and flux calculations on interior faces which can begin right away. The data transfer for inter-processor faces is initiated at the beginning of the right hand side evaluations before volume integrals are calculated. This permits the computation of volume integrals with communication of inter-processor boundary data, thereby, giving DG an edge in-terms of parallel performance. Indeed this was the main reason for the better perfor- 


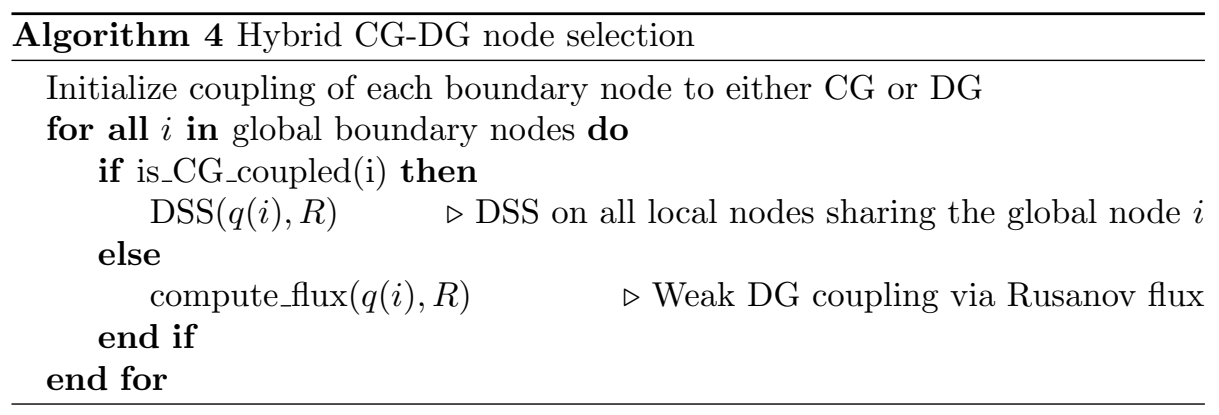

mance of DG compared to CG for the results reported in [21]. We should note that CG can benefit from a similar splitting of the volume integrals into two parts: integration for interior elements and that for elements that have at least one shared inter-processor face. This is currently a work in progress in NUMA. The coupling mechanism for all the CG algorithms is the gather operation which is done implicitly in the case of CGc and explicitly, as part of the DSS operation, in CGd. The gathered data is then communicated to neighboring processors via a synchronous send-receive operation, for instance using MPI_SendRecv. The coupling mechanism for DG is the numerical flux calculated on all interfaces; the values for inter-processor faces are then communicated asynchronously to neighboring processors.

\section{Test cases}

In the following sections, the unified CG/DG implementation is tested against a set of benchmark problems commonly used for benchmarking atmospheric dynamical cores of various scales. The first test case considered, a rising thermal bubble, gauges the cloud resolving capabilities of NUMA with regard to capturing fine-scale structures $[14,13]$. Mesoscale or limited-area modeling capabilities are tested using a density current problem first proposed by Straka et al. [37]. Finally, global scale weather and climate simulation capabilities are tested with an acoustic wave propagation problem proposed in Tomita and Satoh [38]. These problems do not have an analytic solutions so the results for different spatial discretization schemes are compared against one another. However, these tests have sufficiently well understood dynamics that one can discern the correctness of the solution. For example, although we cannot compute error norms for the acoustic wave problem, we can measure the computed acoustic speed and compare against the theoretical speed of sound to discern the proper phase speed of our simulation.

To emphasize the need for stabilization of high-order Galerkin methods in operational mode, we compare results obtained with and without artificial viscosity; other advanced techniques for stabilization can be found in Marras et al. [27]. Different implementations of spatial discretization schemes are compared by measuring the wall clock time taken to complete simulations. We also compare global mass and energy losses calculated as follows. 


$$
\text { Mass loss }=\frac{M(t)-M(0)}{M(0)} \text { where } M(t)=\int_{\Omega} \rho(\mathbf{x}, t) d \Omega
$$

and

$$
\text { Energy loss }=\frac{\xi(t)-\xi(0)}{\xi(0)} \text { where } \xi(t)=\int_{\Omega} E(\mathbf{x}, t) d \Omega
$$

where $E$ is the density total energy of the system.

\subsection{D Rising thermal bubble}

As mentioned in the introduction, current NWP models do not operate with resolutions fine enough to capture characteristics of a cloud. For a model to be considered remotely cloud resolving, the resolution has to be at least $1 \mathrm{~km}$. The following test case is conducted with a resolution of $25 \mathrm{~m}$, and thus, can be considered to be cloud resolving. In the following, we consider the evolution of a warm bubble [14] in a neutrally stratified atmosphere of constant potential temperature $\theta_{0}$.

The initial conditions are in hydrostatic balance where pressure decreases with height as

$$
p=p_{0}\left(1-\frac{g z}{c_{p} \theta_{0}}\right)^{c_{p} / R} .
$$

The potential temperature perturbation is given by

$$
\theta^{\prime}= \begin{cases}0 & \text { for } r>r_{c} \\ \frac{\theta_{c}}{2}\left(1+\cos \left(\frac{\pi r}{r_{c}}\right)\right) & \text { for } r \leq r_{c}\end{cases}
$$

where

$$
r=\sqrt{\left(x-x_{c}\right)^{2}+\left(z-z_{c}\right)^{2}} .
$$

We run the rising thermal bubble simulation on a $[0 \mathrm{~m}, 1000 \mathrm{~m}]^{3}$ domain, with $\left(x_{c}, z_{c}\right)=(500 \mathrm{~m}, 350 \mathrm{~m}), r_{c}=250 \mathrm{~m}$, and $\theta_{c}=0.5 \mathrm{~K}, \theta_{0}=300 \mathrm{~K}$. The domain is subdivided into $10 \times 1 \times 10$ elements (in $x, y$, and $z$, respectively) with polynomial order $N=4$ set in all directions for a total of about 180 k nodes. The effective resolution of the model is about $25 \mathrm{~m}$. The simulation is run for 1000s with a constant time step of $\Delta t=0.02 \mathrm{~s}$ using an explicit fourth order Runge-Kutta method for time integration. A no-flux boundary condition is used at all boundaries.

First, we run CG and DG without artificial diffusion. The results for these simulations are shown in Figs. 3 and 4. After 300 seconds, the bubble starts to become unstable highlighting the need for stabilization for both CG and DG. The plots also show that all the implemented CG flavors, namely CGc, CGdseparate and CGd-unified, yield almost identical results at all times. DG also gives results identical to $\mathrm{CG}$ before instability sets in after which the results are 
distinctly different from CG's. This is attributed to the fact that DG has an additional dissipation mechanism through its use of an upwind-biased flux, i.e., the Rusanov flux in the case of NUMA. The hybrid CGDG version uses DG in the upper half $(z>500 m)$ and CG in the bottom half. We can see that the results of the hybrid version overlaps with that of DG even at $t=400 \mathrm{sec}$ where DG showed differences with CG.

The second group of plots in Figs. 5 and 6 show results for simulations run with an artificial viscosity of $\nu=1.5 \mathrm{~m}^{2} / \mathrm{s}$. Comparing the results against the previous results, we can clearly see that artificial diffusion has improved the results at 400 and 500 seconds significantly. Unlike in the previous simulations, this time DG also gives identical results to the different CG flavors at all times.

The most important metric we are interested in is the wall clock times of different implementations, shown in Tables 1 and 2. The simulations are run using 16 MPI threads on a shared memory computer. The fastest implementation is CGc which uses a global node storage scheme. We have mentioned in the introduction that CGc results in about 1.3 times fewer degrees of freedom than DG for the degree of polynomials used in NUMA; the ratio is 1.56 for this particular case. DG and CGd-separate ${ }^{7}$ come in second. This is attributed to the fact that in places where DG does flux calculations, CGd-separate does the DSS operation; the rest of the code is shared by all the methods. Evidently these two operations result in comparable number of operations which is the reason why the wall clock times for the runs are similar. This is no surprise because the numerical flux calculation process using Rusanov's method can be thought of as a similar process as the averaging in the DSS operation especially when no upwinding term is used in the former. As noted before, these two processes are necessary for coupling the otherwise decoupled system of equations in the respective methods. The cost of communication at ghost cells in the parallel implementation is also similar in both methods. The slowest implementation is CGd-unified that uses the DG formulation for setting no-flux boundary conditions on top of that required for imposing essential boundary conditions using CG's way. As a result, we expected CGd-unified to be slightly slower than CGd-separate; however, it is possible to skip the CG way of imposing boundary conditions in CGd-unified and bring it up to speed with CG-separate.

The wall clock times for the simulations run with artificial viscosity show that the explicit Laplacian operator in Eq. (9) adds a significant cost; however, the observations we made in the previous paragraph regarding the relative performance of different implementations remain valid. On average, CGc is about $11 \%$ faster than CGd-separate and CGd-unified, which in turn are about $7 \%$ faster than DG.

The next metric we use to compare the different implementations is the global mass and energy losses. The equations set used for these simulations, namely Set2C, is mass-conserving by construction, therefore, the mass loss metric is a measure of numerical error. Table 1 and 2 show that mass is conserved

\footnotetext{
${ }^{7} \mathrm{CGd}$-separate is similar to CGc except for its use of local element wise storage.
} 


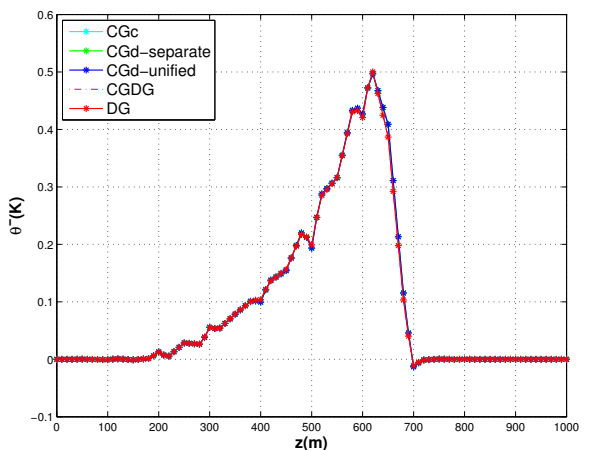

(a) $300 \mathrm{sec}$

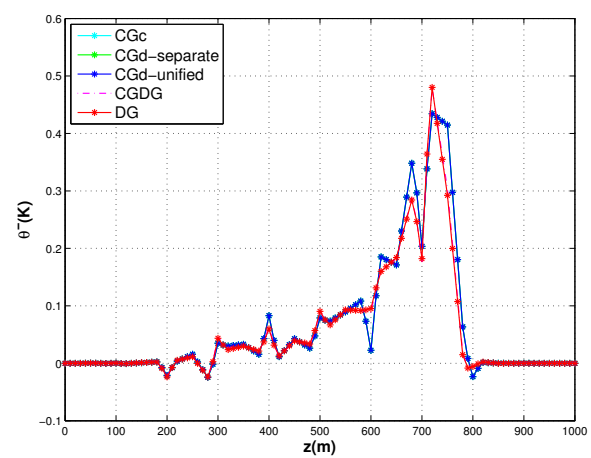

(b) $400 \mathrm{sec}$

Figure 3: Potential temperature perturbation $\theta^{\prime}(K)$ along the middle vertical section $\mathrm{x}=500 \mathrm{~m}$ for the 2D rising thermal bubble problem. Results for CGc, CGd-separate, CGd-unified, CGDG and DG implementations are shown at $\mathrm{t}=300$ and 400 seconds. A grid of $10 \times 1 \times 10$ elements with 4th degree polynomials is used. No filters or artificial viscosity is used for these simulations.

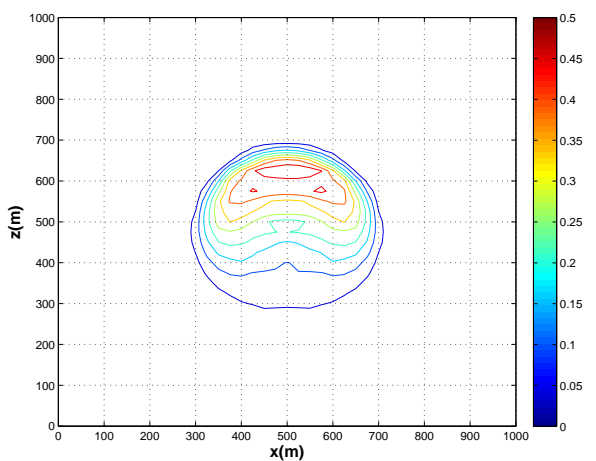

(a) $300 \mathrm{sec}$

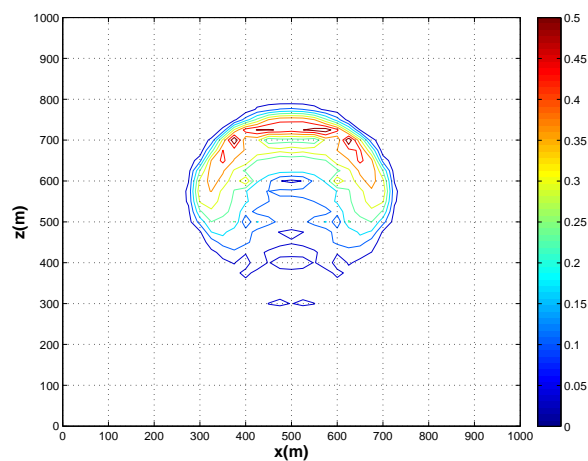

(b) $400 \mathrm{sec}$

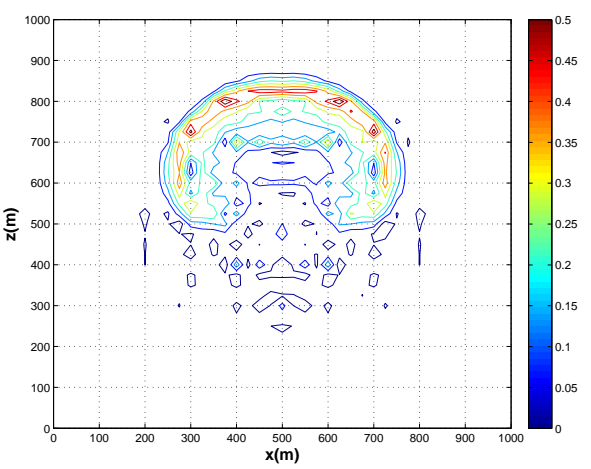

(c) $500 \mathrm{sec}$

Figure 4: Potential temperature perturbation $\theta^{\prime}(K)$ contour plot for the 2D rising thermal bubble problem run using CGc. Results are shown at $\mathrm{t}=300,400$ and 500 seconds. 


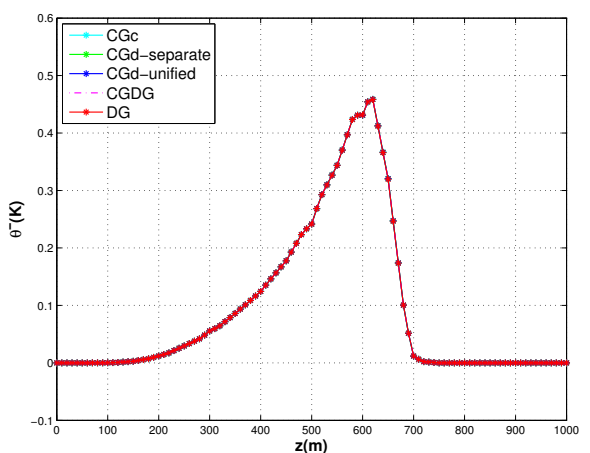

(a) $300 \mathrm{sec}$

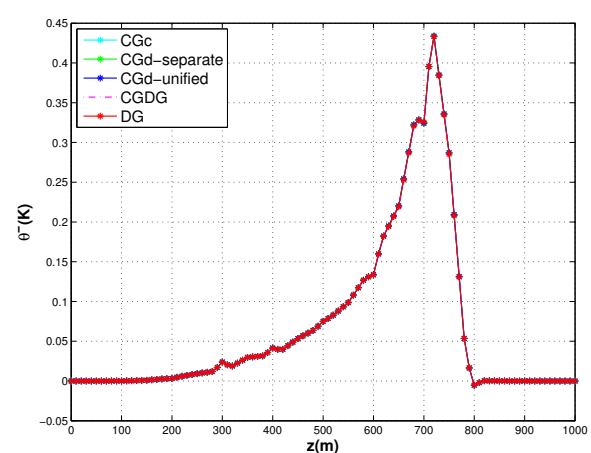

(b) $400 \mathrm{sec}$

Figure 5: Potential temperature perturbation $\theta^{\prime}(K)$ along the middle vertical section $\mathrm{x}=500 \mathrm{~m}$ for the 2D rising thermal bubble problem with an artificial viscosity of $\nu=1.5 \mathrm{~m}^{2} / \mathrm{s}$ for stabilization. Results for CGc, CGd-separate, CGd-unified, CGDG and DG implementations are shown at $\mathrm{t}=300$ and 400 seconds. A grid of $10 \times 1 \times 10$ elements with 4 th degree polynomials is used.

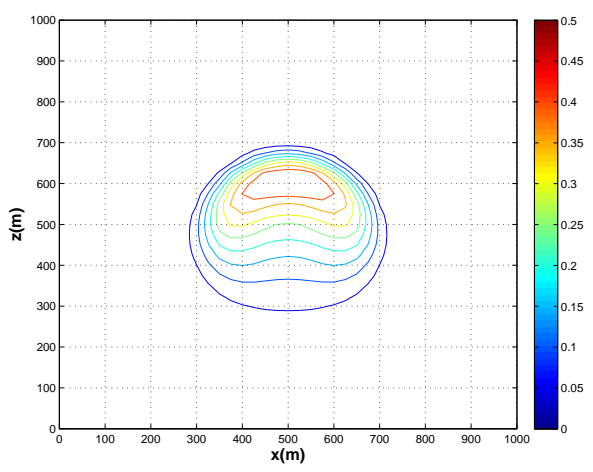

(a) $300 \mathrm{sec}$

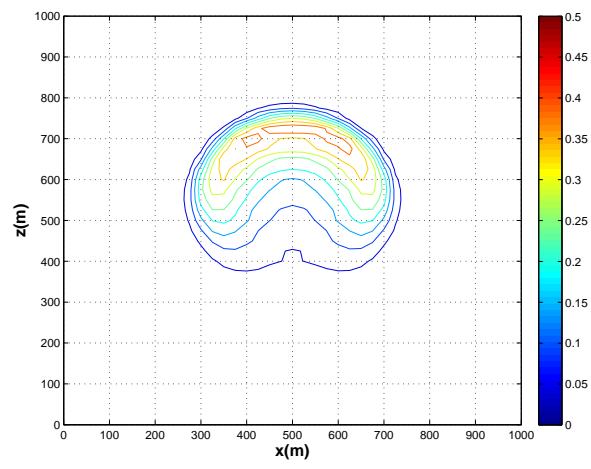

(b) $400 \mathrm{sec}$

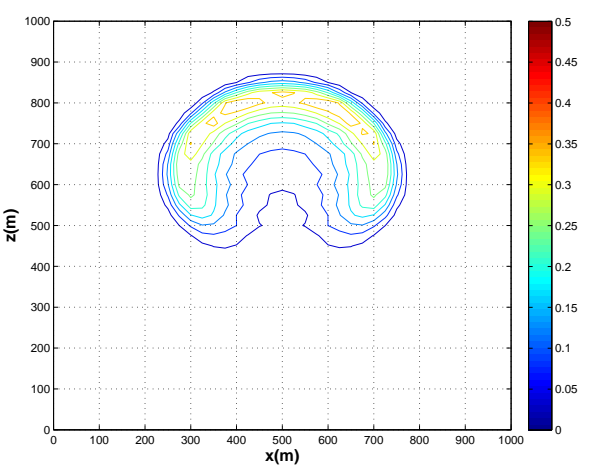

(c) $500 \mathrm{sec}$

Figure 6: Potential temperature perturbation $\theta^{\prime}(K)$ contour plot for the 2D rising thermal bubble problem run with CGc and an artificial viscosity of $\nu=1.5 \mathrm{~m}^{2} / \mathrm{s}$ for stabilization. Results are shown at $\mathrm{t}=300,400$ and 500 seconds. 
Table 1: Comparison between different implementations for a 500s run of the 2D rising thermal bubble simulation.

\begin{tabular}{llrl}
\hline Method & Wall clock time(s) & Mass loss & Energy loss \\
\hline CGc & 89.79 & $1.33734945 \mathrm{E}-16$ & $1.01843549 \mathrm{E}-08$ \\
CGd-separate & 110.18 & $1.33734945 \mathrm{E}-16$ & $1.01843549 \mathrm{E}-08$ \\
CGd-unified & 118.49 & $1.33734945 \mathrm{E}-16$ & $1.01843549 \mathrm{E}-08$ \\
DG & 109.23 & $2.67469890 \mathrm{E}-16$ & $1.08277315 \mathrm{E}-09$ \\
CGDG & 113.24 & $1.33734945 \mathrm{E}-16$ & $2.64740380 \mathrm{E}-09$ \\
\hline
\end{tabular}

Table 2: Comparison between different implementations for a 500s run of the 2D rising thermal bubble simulation with artificial viscosity.

\begin{tabular}{llrl}
\hline Method & Wall clock time(s) & Mass loss & Energy loss \\
\hline CGc & 130.31 & $1.33734945 \mathrm{E}-16$ & $7.98369926 \mathrm{E}-08$ \\
CGd-separate & 150.69 & $1.33734945 \mathrm{E}-16$ & $7.98369926 \mathrm{E}-08$ \\
CGd-unified & 157.66 & $1.33734945 \mathrm{E}-16$ & $7.98369925 \mathrm{E}-08$ \\
DG & 151.27 & $2.67469890 \mathrm{E}-16$ & $7.93205596 \mathrm{E}-08$ \\
CGDG & 159.87 & $0.00000000 \mathrm{E}-00$ & $7.93039150 \mathrm{E}-08$ \\
\hline
\end{tabular}

up to machine precision by all methods. Energy is not conserved by any of the methods because Set2C is formulated using potential temperature instead of total energy and so this equation set is not meant to conserve energy. ${ }^{8}$ The slightly higher energy loss in Table 4 is due to the use of artificial diffusion for the momentum and energy equations. We chose not to apply artificial diffusion to the continuity equation, and as result, no degradation in mass conservation is observed.

To demonstrate the advantage of CGd-unified over CGd-separate, we rerun the rising thermal bubble problem on a domain with a hill of shape defined as

$$
h(x)=h_{m} \cos ^{2}\left(\frac{\pi}{2} \frac{\left(x-x_{c}\right)}{a_{c}}\right)
$$

where $h_{m}=100 \mathrm{~m}$ is the height, $a_{c}=100 \mathrm{~m}$ is the half width, $\left(x_{c}, z_{c}\right)=$ $(50 \mathrm{~m}, 0 \mathrm{~m})$ is the central location of the hill. The results for this test case are summarized in Table 3. The mass and energy conservation properties of CGc and CGd-separate have greatly suffered from the addition of the hill. On the other hand, CGd-unified maintains its mass and energy conservation properties as before the hill was added, therefore, the reason must be in the different way the NFBCs are applied for CG and DG as explained in Section 3.3. DG also maintains its mass conservation properties further consolidating the validity of this observation. It should be noted that the degradation in conservation observed for CGc and CGd-separate can be avoided if special precautions are taken. Nonetheless, we show these results to make the point that the DG-type

\footnotetext{
${ }^{8}$ Energy can only be conserved for this equation set up to time-truncation error.
} 
Table 3: Comparison between different implementations for a 500s run of the 2D rising thermal bubble simulation over a hill.

\begin{tabular}{llrl}
\hline Method & Wall clock time(s) & Mass loss & Energy loss \\
\hline CGc & 129.13 & $1.63220006 \mathrm{E}-06$ & $2.37663619 \mathrm{E}-06$ \\
CGd-separate & 151.77 & $1.63220009 \mathrm{E}-06$ & $2.37663623 \mathrm{E}-06$ \\
CGd-unified & 160.16 & $0.00000000 \mathrm{E}-00$ & $8.91492998 \mathrm{E}-08$ \\
DG & 149.74 & $1.35138469 \mathrm{E}-16$ & $8.68371202 \mathrm{E}-08$ \\
\hline
\end{tabular}

boundary condition enforcement handles orography quite naturally without any special precautions.

\subsection{Density current}

To test the mesoscale modeling capabilities of NUMA, we use the density current problem first proposed in [37]. This test case concerns the evolution of a cold bubble in a neutrally stratified atmosphere of constant potential temperature $\theta_{0}$. Unlike the rising thermal bubble problem of the previous section, the bubble used here is colder than the surrounding air. As a result, the bubble first sinks to the ground and then moves along the ground while forming vortices due to Kelvin-Helmholtz instabilities. The numerical solution of this problem using high order methods requires using artificial diffusion or other stabilization techniques. We use an artificial viscosity of $\nu=75 \mathrm{~m}^{2} / \mathrm{s}$ for this work as proposed in [37].

Aside from the difference in geometry and bubble temperature, the problem setup is similar to that of the rising thermal bubble test case. The computational domain for this problem is $\Omega=[0,25600 \mathrm{~m}] \times[0, \infty] \times[0,6400 \mathrm{~m}]$. The background state is isothermal with an initial potential temperature of $\theta_{0}=300$ $\mathrm{K}$, and the potential temperature perturbation is given by Eq. (22) where $\theta_{c}=-$ $15 \mathrm{~K}$. The bubble is ellipsoidal with radii $\left(r_{x}, r_{z}\right)=(4000 \mathrm{~m}, 2000 \mathrm{~m})$ and centered at $\left(x_{c}, z_{c}\right)=(0,3000 \mathrm{~m})$. The computational domain is subdivided into $128 \times 1$ x 32 elements with polynomial order $N=4$ set in all directions for an effective resolution of $50 \mathrm{~m}$. Free slip boundary conditions are used for all boundaries.

Figure 7 shows the evolution of the bubble every 300 seconds up to 900 seconds. The transport and diffusion of potential temperature perturbation is evident as the bubble drops to the ground due to gravity and then rolls over the ground forming vortices. The vortical structures formed at $\mathrm{t}=900 \mathrm{sec}$ are similar to that reported in [37]. The location of the front at $900 \mathrm{sec}$ is about $14720 \mathrm{~m}$ for our simulations. The results reported in [37] for different methods using the same resolution and artificial viscosity range from $14400 \mathrm{~m}$ to $15000 \mathrm{~m}$, therefore, our results are within the acceptable range.

Next, we make comparisons between different CG and DG implementations of NUMA by plotting the potential temperature perturbation along a line in the $\mathrm{x}$-direction and at a height of $1800 \mathrm{~m}$. As Fig. 8 shows, no discernible differences are observed between different implementations of CG and DG. This is attributed to the fact that the artificial viscosity needed to stabilize this 
simulation is relatively high, which dominates any differences CG and DG have regarding stability. Figure 9 shows the mass and energy losses incurred by the different implementations. This case is typically run for $900 \mathrm{sec}$ but we let the wave hit the other end of the wall by running the simulation for 5400 seconds. We observe that CGc and CGd-separate show identical mass and energy loss properties at all time steps. CGd-unified shows differences with CGc in this regard due to the different way no flux boundary conditions are imposed. The hybrid CGDG simulation uses DG in the bottom half of the domain $(z<3000 \mathrm{~m}$ ) and CG in the upper half.

\subsection{Acoustic wave on the sphere}

As a verification of the global scale atmospheric dynamics capabilities of NUMA, we consider the case of an acoustic wave traveling around the globe described in [38]. The initial state for this problem is hydrostatically balanced with an isothermal background potential temperature of $\theta_{0}=300 \mathrm{~K}$. A perturbation pressure $p^{\prime}$ is superimposed on the reference pressure

$$
p^{\prime}=f(\lambda, \phi) g(r)
$$

where

$$
\begin{aligned}
f(\lambda, \phi)= \begin{cases}0 & \text { for } r>r_{c} \\
\frac{\Delta P}{2}\left(1+\cos \left(\frac{\pi r}{r_{c}}\right)\right) & \text { for } r \leq r_{c}\end{cases} \\
\\
g(r)=\sin \left(\frac{n_{v} \pi r}{r_{T}}\right)
\end{aligned}
$$

where $\Delta P=100 \mathrm{~Pa}, n_{v}=1, r_{c}=r_{e} / 3$ is one third of the radius of the earth $r_{e}=6371 \mathrm{~km}$ and a model altitude (top) of $r_{T}=10 \mathrm{~km}$. The geodesic distance $r$ is calculated as

$$
r=r_{e} \cos ^{-1}\left[\sin \phi_{0} \sin \phi+\cos \phi_{0} \cos \phi \cos \left(\lambda-\lambda_{0}\right)\right]
$$

where $\left(\lambda_{0}, \phi_{0}\right)$ is the origin of the acoustic wave.

A cubed sphere grid of $10 \times 10 \times 3$ elements with $3^{\text {rd }}$ order polynomials is used. ${ }^{9}$ No-flux boundary conditions are used at the bottom and top surfaces. First, we run CG and DG without using any stabilization methods. The results for this case, illustrated in the top row of Fig. 11, show that all CG methods need some sort of stabilization. On the other hand, DG gives a non-oscillatory result due to its use of upwind-biased numerical flux. The dissipation in DG is evident from the decreased size and intensity of the inner ring. Visual comparison of plots showing the location of the wave at different hours, shown in Fig. 10, against results in [38] indicate that the results are quite similar.

\footnotetext{
${ }^{9}$ The cubed sphere grid comprised of $10 \times 10 \times 3$ elements will have a total of $6 \times 10 \times 10 \times 3=$ 1800 elements where the 6 is due to the six faces of the cubed sphere.
} 


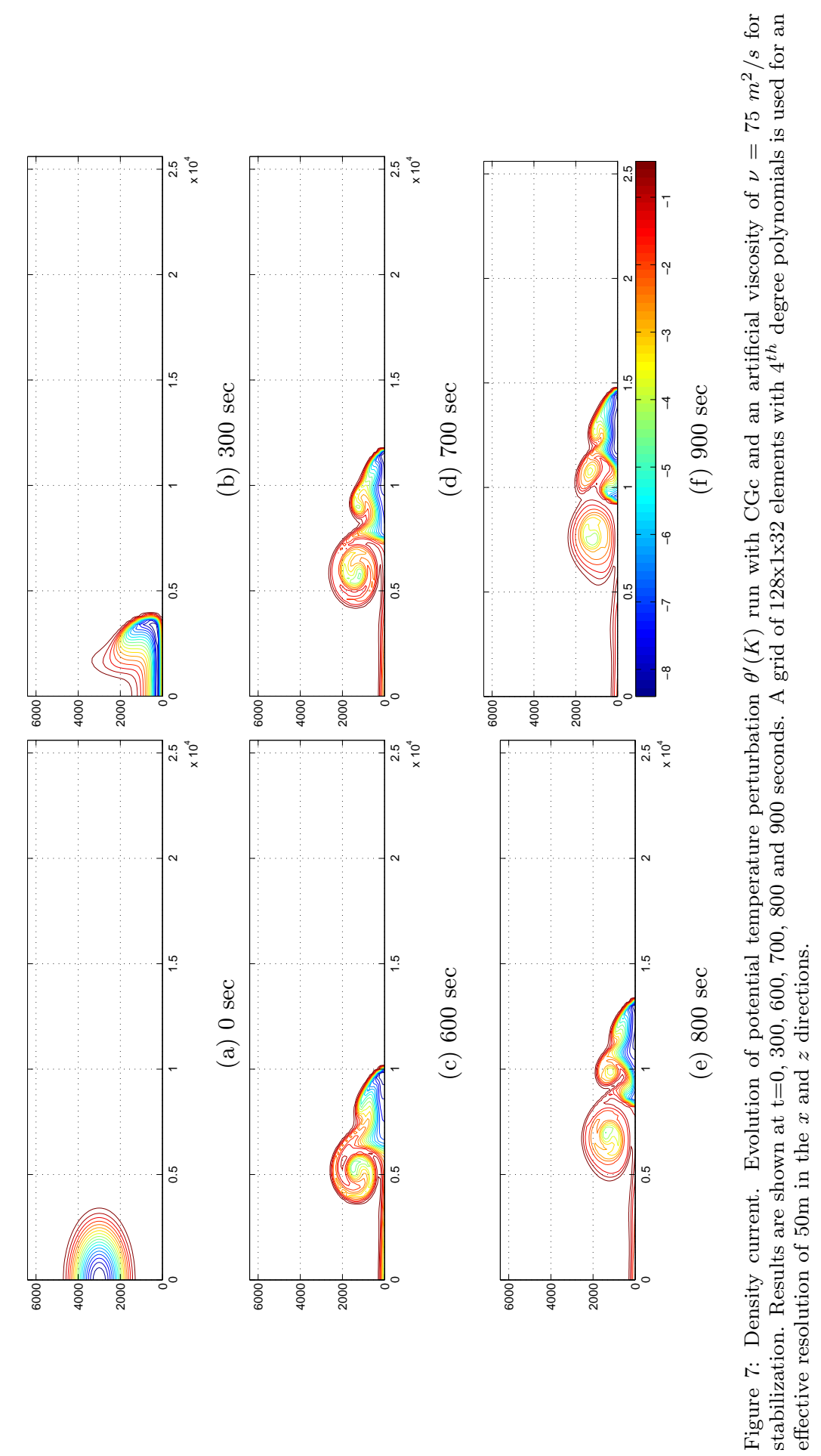



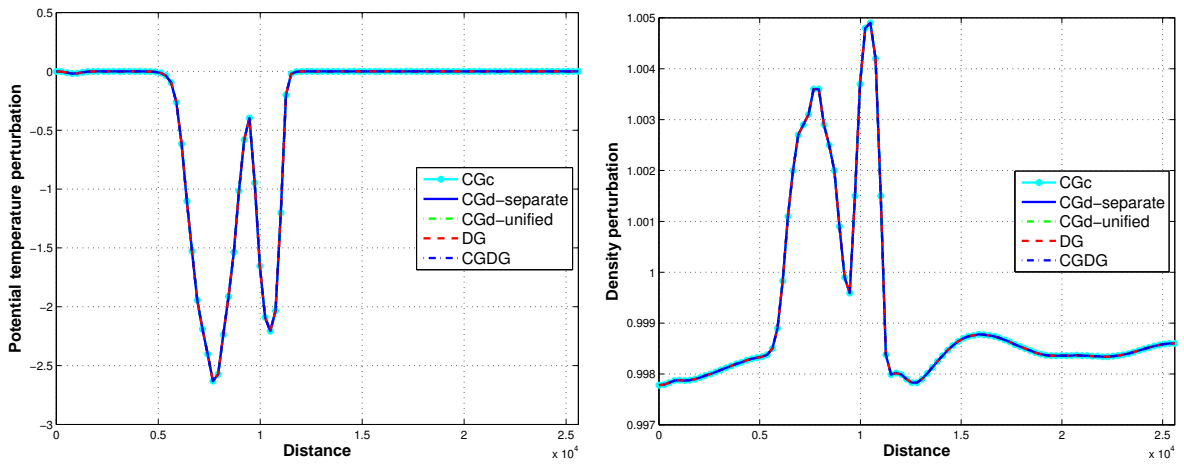

Figure 8: Density current. Comparison of potential temperature and density perturbations between different methods. The plots show values of $\theta^{\prime}$ at 900 seconds along a line in the $\mathrm{x}$-direction and a height of $1800 \mathrm{~m}$. The results for all implementations are identical. The artificial viscosity used for this problem, $\nu=75 \mathrm{~m}^{2} / \mathrm{s}$, is relatively high and this suppresses differences CG and DG may have.
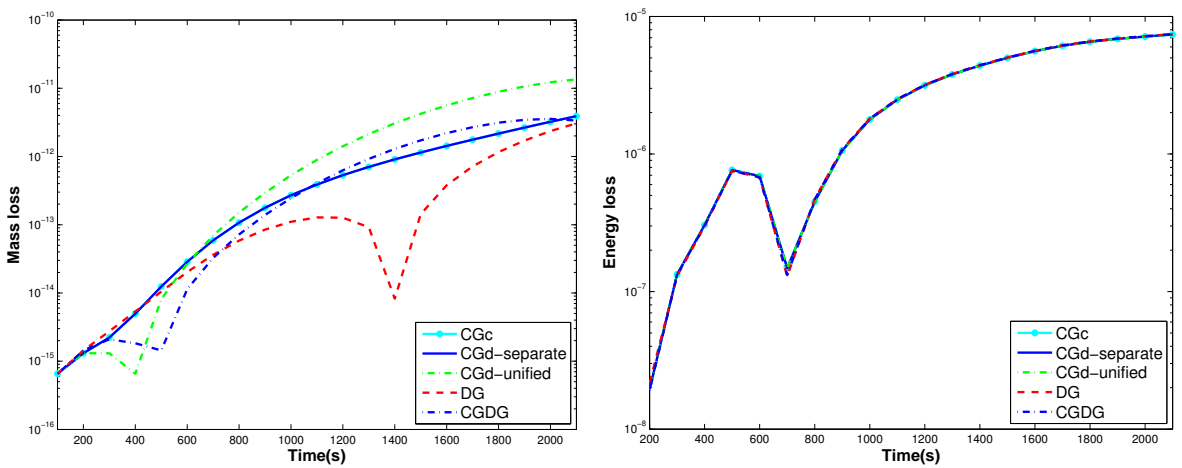

Figure 9: Density current. Comparison of mass and energy losses for the density current problem run up to 2000 seconds. A grid resolution of $50 \mathrm{~m}$ and artificial viscosity of $\nu=75$ $\mathrm{m}^{2} / \mathrm{s}$ is used. 
Table 4: Comparison between different implementations for a 1 hour run of an acoustic wave on a sphere simulation.

\begin{tabular}{llrl}
\hline Method & Wall clock time(s) & Mass loss & Energy loss \\
\hline CGc & 613.81 & $1.1571216 \mathrm{E}-14$ & $1.1970014 \mathrm{E}-10$ \\
CGd-separate & 780.00 & $1.1571216 \mathrm{E}-14$ & $1.1970014 \mathrm{E}-10$ \\
CGd-unified & 801.60 & $7.0873696 \mathrm{E}-15$ & $1.1970014 \mathrm{E}-10$ \\
DG & 892.88 & $1.1571216 \mathrm{E}-14$ & $1.5413990 \mathrm{E}-10$ \\
CGDG & 947.65 & $9.4450047 \mathrm{E}-14$ & $1.5594926 \mathrm{E}-10$ \\
\hline
\end{tabular}

In the previous sections, we used artificial diffusion for stabilization; here we use filters instead, namely, the Boyd-Vandeven spatial filter [4]. The description of the filtering process in NUMA for both CG and DG can be found in [14]. The results for this case are shown in the bottom row of Fig. 11. Clearly use of filters helps to improve results for all CG methods. Also, the size and intensity of the inner ring have decreased due to the dissipation introduced by the filters. Numerical comparison of the different methods is shown in Fig. 11 along a geodesic line starting from the origin of the acoustic wave extending to the antipode. We can see that all CG and DG implementations give nearly identical results except for the case where filters are used; the result with filters show some dispersion. CG with filters and DG without it gave comparably smooth results for this test case. We observe that towards the tail of the plots, where spurious oscillations are observed for the unstabilized CG methods, the explicit filter operation applied to CGc and the implicit filter of DG both have removed high frequency waves and result in smooth solutions.

The wall clock time comparison after 1 hour of the acoustic wave simulation is shown in Table. 4. The relative performance of different implementations is more or less the same as before, however, CGc is faster by a bigger margin for this case because the ratio of the number of grid points of DG to CG is about 1.5. Here also, the mass conservation is good for all spatial discretization methods including the hybrid CGDG version, which was tested using CG in the lower half of the sphere $(z<0)$ and DG in the upper half $(z>0)$. Figure 12 compares the density perturbation between the different methods. The CG area in the hybrid CGDG simulation behaves the same way (shows oscillations) as the pure CG version when filters are not used. 


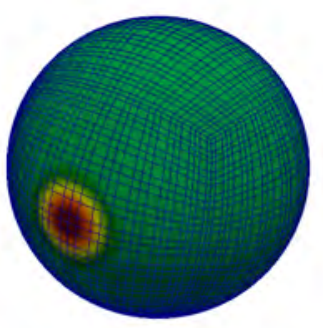

(a) $0 \mathrm{~h}$

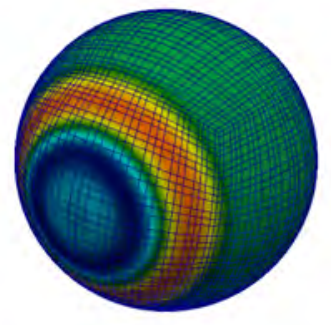

(b) $4 \mathrm{~h}$

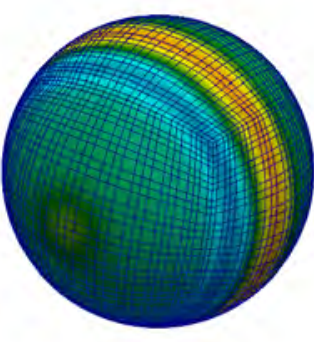

(c) $7 \mathrm{~h}$

Density perturbation

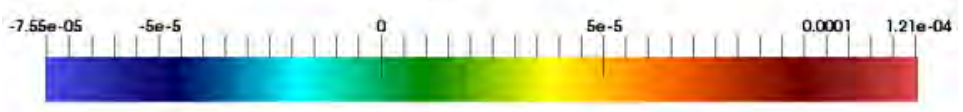

Figure 10: Propagation of an acoustic wave. The density perturbation after 0 hour, 4 hours and 7 hours. A cubed sphere grid with 10x10 elements in the horizontal and 3 elements in the vertical with 3 rd degree polynomial is used. 


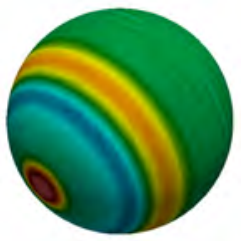

(a) $\mathrm{CGc}$

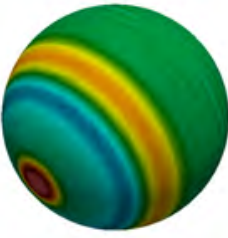

(b) CGd-separate

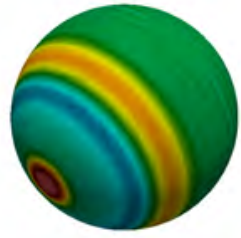

(c) CGd-unified

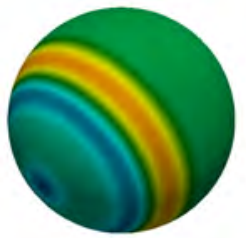

(d) DG

\section{Density perturbation}

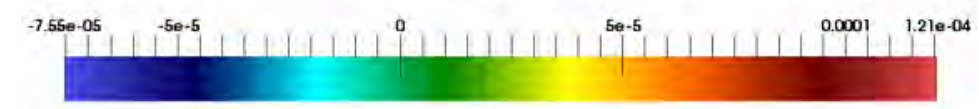

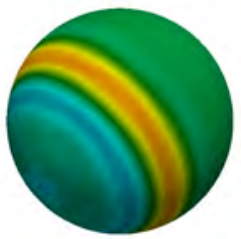

(e) CGc-filter

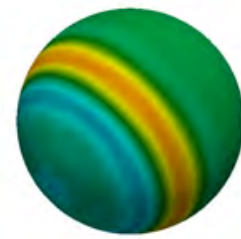

(f) CGd-sep-filter

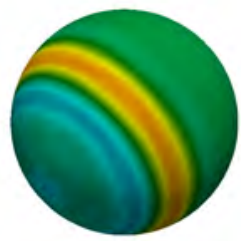

(g) CGd-unif-filter

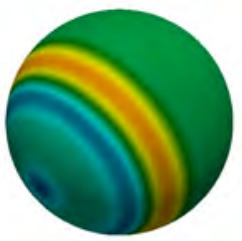

(h) DG-filter

Figure 11: Propagation of an acoustic wave. The density perturbation after 5 hours is shown for CGc, CGd-separate, CGd-unified, and DG. The top rows show results for simulations run without using filters for stabilization; and the bottom show results for simulations run with a filter. The plots suggest that all CG implementations need stabilization; on the other hand DG yielded a smooth result through its use of an upwind-biased flux. A cubed sphere grid with $10 \times 10$ elements in the horizontal and 3 elements in the vertical with $3^{\text {rd }}$ degree polynomial is used.

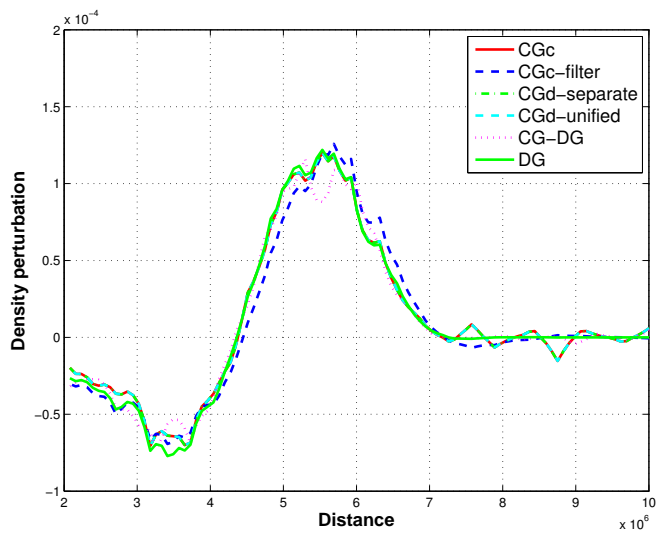

Figure 12: Comparison of density perturbation between different methods for the acoustic wave propagation problem after 4 hours. The horizontal axis is the geodesic distance from the origin of the acoustic wave to its antipode. The CGc-filter method used a filter for stabilization. 


\section{Conclusions}

We have presented a unified continuous and discontinuous Galerkin approach for the numerical solution of the 3D Euler equations and implemented it in the Non-hydrostatic Unified Model of the Atmosphere. The motive for unifying CG into the DG framework is that the latter possesses several desirable properties that may make it more robust than CG for Numerical Weather Prediction; by more robust here we mean more stable (as we saw in the acoustic wave test) and more straightforward to implement with certain types of boundary conditions (as we saw with the rising thermal bubble problem with an embedded hill). Classical CG is implemented using a global storage scheme so that the required $C^{0}$ continuity is satisfied during construction (assembly) of the mass matrix and right hand side vector. If we wish to incorporate $\mathrm{CG}$ within the DG framework, we have to use a local storage scheme, in the spirit of DG, that allows for discontinuities at element interfaces. To get a $C^{0}$ continuous solution afterwards, an equality constraint has to be imposed on the duplicate nodes at element interfaces to make them effectively one node.

First, we presented several constraint imposition methods borrowed from the finite element literature to expose the conversion process from the DG to CG formulations as a constraint imposition process. Then, we showed how Direct Stiffness Summation (DSS) can be applied for the special case of a diagonal mass matrix, and then implemented it in NUMA. DSS gathers values from all slave nodes sharing the same global identification number and then scatters back the sum total value back to the slave nodes. This way DG and CG share the same code except in places where DG computes fluxes at element interfaces CG does the DSS operation. We were also able to retain the classical CG implementation using a global storage scheme (CGc) within the same framework using a simple idea of indexing that switches between one-to-one or many-to-one mapping of global to local nodes for DG and CG respectively. The value of going through this exercise is that all of the attributes of a DG model (e.g., non-conforming adaptive mesh refinement, the use of limiters, etc.) can be included into a classical CGc model using our approach. The unification also allowed us to include a hybrid CGDG method, which uses DG in some regions, e.g. where the flow is non-smooth, and CG in others. The hybrid approach can be dynamically tuned in accordance with the flow regime.

The different implementations of CG and DG are tested using benchmark test cases that have properties representative of typical atmospheric dynamics: the rising thermal bubble problem [14] for testing cloud-resolving capabilities of resolution less than $1 \mathrm{~km}$, the density current problem for testing the mesoscale modeling capability [37], and finally an acoustic wave propagation on the sphere [38] problem for testing the global circulation modeling capability. The results obtained from all the test cases yielded identical results for all the CG implementations; DG also gave similar results to the CG methods when the latter are stabilized using artificial viscosity or filters. 


\section{Acknowledgment}

The authors gratefully acknowledge the support of the Office of Naval Research through program element PE-0602435N.

\section{References}

[1] Arnold, D., Brezzi, F., Cockburn, B., Marini, L., 2005. Unified analysis of discontinuous galerkin methods for elliptic problems. J. Sci. Comput. $22 / 23,25-45$.

[2] Bagheri, B., Scott, L. R., Zhang, S., 1994. Special issue selection of papers presented at icosahom'92 implementing and using high-order finite element methods. Finite Elements in Analysis and Design 16 (3), 175 - 189.

[3] Bernardi, C., Maday, Y., Rapetti, F., 2005. Basics and some applications of the mortar element method. GAMM-Mitteilungen 28 (2), 97-123.

[4] Boyd, J., 1996. The erfc-log filter and the asymptotics of the Euler and Vandeven sequence accelerations. In: Houston Journal of Mathematics. Proceedings of the third International Conference on Spectral and High Order Methods. pp. $267-276$.

[5] Boyd, J., 1998. Two comments on filtering for chebyshev and legendre spectral and spectral element methods. J. Comput. Phys. 143, $283-288$.

[6] Cangiani, A., Chapman, J., Georgoulis, E., Jensen, M., 2013. On the stability of continuous-discontinuous galerkin methods for advection?diffusion?reaction problems. Journal of Scientific Computing 57 (2), 313-330.

[7] Cockburn, B., Gopalakrishnan, J., Lazarov, R., 2009. Unified hybridization of discontinuous Galerkin, mixed and continuous Galerkin methods for second order elliptic problems. SIAM J. Num. Anal. 47, 1319 - 1365.

[8] Dawson, C., Proft, J., 2002. Coupling of continuous and discontinuous galerkin methods for transport problems. Computer Methods in Applied Mechanics and Engineering 191 (29?30), 3213 - 3231.

[9] Dongarra, J., Strohmaier, E., Simon, H., Meuer, H., 2015. Top500.org. URL http://www.top500.org/lists/2015/06/

[10] Felippa, C., 1977. Error analysis of penalty function techniques for constraint definition in linear algebraic systems. International J. Num. Methods. in Engg. 11, $709-728$.

[11] Fischer, P., Mullen, J., 2001. Filter-based stabilization of spectral element methods. Comptes Rendus del lAcademie des Sciences - Series I - Mathematics, 265-270. 
[12] Fournier, A., Taylor, M., Tribbia, J., MAR 2004. The spectral element atmosphere model (SEAM): High-resolution parallel computation and localized resolution of regional dynamics. Monthly Weather Review 132 (3), $726-748$.

[13] Giraldo, F. X., Kelly, J. F., Constantinescu, E. M., 2013. Implicit explicit formulations of a three dimensional non-hydrostatic unified model of the atmosphere (numa). SIAM J. Sci. Comput. 35, 1162 - 1194.

[14] Giraldo, F. X., Restelli, M., 2008. A study of spectral element and discontinuous galerkin methods for the navier-stokes equations in nonhydrostatic mesoscale atmospheric modeling: Equation sets and test cases. J. Comput. Phys. 227, $3849-3877$.

[15] Giraldo, F. X., Restelli, M., 2010. High-order semi-implicit time-integrators for a triangular discontinuous galerkin oceanic shallow water model. Int. J. Numer. Meth. Fl. 63, 1077-1102.

[16] Giraldo, F. X., Restelli, M., Laeuter, M., 2010. Semi-implicit formulations of the Navier-Stokes equations: application to nonhydrostatic atmospheric modeling. SIAM Journal on Scientific Computing 32 (6), 3394-3425.

[17] Giraldo, F. X., Rosmond, T. E., JAN 2004. A scalable spectral element eulerian atmospheric model (SEE-AM) for NWP: Dynamical core tests. Monthly Weather Review 132 (1), 133-153.

[18] Hestenes, M., 1969. Multiplier and gradient methods. Journal of Optimization Theory and Applications 4 (5), 303-320.

[19] Hindenlang, F., Gassner, G. J., Altmann, C., Beck, A., Staudenmaier, M., Munz, C.-D., 2012. Explicit discontinuous galerkin methods for unsteady problems. Computers \& Fluids 61, $86-93$.

[20] Jablonowski, C., Williamson, D., 2011. The pros and cons of diffusion, filters and fixers in atmospheric general circulation models. In: Numerical techniques for Global atmospheric models. Vol. 80 of Lecture notes in Computational Science and Engineering. pp. $381-482$.

[21] Kelly, J. F., Giraldo, F. X., 2012. Continuous and discontinuous galerkin methods for a scalable three-dimensional nonhydrostatic atmospheric model: limited area mode. J. Comput. Phys. 231, $7988-8008$.

[22] Kirby, R., Knepley, M., Scott, L. R., 2004. Evaluation of the action of finite element operators. Tech. rep.

[23] Kirby, R., Sherwin, S., Cockburn, B., 2012. To cg or to hdg: A comparative study. Journal of Scientific Computing 51 (1), 183-212.

[24] Klöckner, A., Warburton, T., Bridge, J., Hesthaven, J., 2009. Nodal discontinuous galerkin methods on graphics processors. Journal of Computational Physics 228 (21), $7863-7882$. 
[25] Kopera, M. A., Giraldo, F. X., 2015. Mass conservation of unified continuous and discontinuous element-based galerkin methods on dynamically adaptive grids with application to atmospheric simulations. J. Comput. Phys. accepted, 000-000.

[26] Lions, J., 1968. Problemes aux limites non homogenesa donées irrégulieres: Une méthode d?approximation. Numerical Analysis of Partial Differential Equations (CIME 2 Ciclo, Ispra, 1967), 283-292.

[27] Marras, S., Kelly, J., Giraldo, F., Vazquez, M., 2012. Variational multiscale stabilization of high-order spectral elements for the advection?diffusion equation. J. Comput. Phys. 231, $7187-7213$.

[28] Mavriplis, C., 1994. Adaptive mesh strategies for the spectral element method. Computer Methods in Applied Mechanics and Engineering 116 (1?4), $77-86$.

[29] Müller, A., Kopera, M., Marras, S., Wilcox, L., Isaac, T., Giraldo, F., 2016. Strong scaling for numerical weather prediction at petascale with the atmospheric model numa. Submitted to : 30th IEEE International Parallel and Distributed Processing Symposium.

[30] Nair, R., Choi, H., Tufo, H., 2009. Computatinal aspects of a scalable highorder discontinuous Galerkin atmospheric dynamical core. Comput. Fl. 38, $309-319$.

[31] Nguyen, N., Peraire, J., 2012. Hybridizable discontinuous galerkin methods for partial differential equations in continuum mechanics. J. Comput. Phys. $231,5955-5988$.

[32] Nguyen, N., Peraire, J., Cockburn, B., 2009. An implicit high-order hybridizable discontinuous galerkin method for nonlinear convectiondiffusion equations. Journal of Computational Physics 228 (23), 8841 - 8855.

[33] NOAA, 2015. High performance computing plan 2015-2020. National Oceanic and Atmospheric Adminstration, 1 - 11.

[34] Paz, M., Leigh, W., 2001. Static condensation and substructuring. In: Integrated Matrix Analysis of Structures. Springer US, pp. 239-260.

[35] Rivire, B., 2008. Discontinuous Galerkin Methods for Solving Elliptic and Parabolic Equations. Society for Industrial and Applied Mathematics.

[36] Rosenberg, D., Fournier, A., Fischer, P., Pouquet, A., 2006. Geophysical astrophysical spectral-element adaptive refinement (gaspar): Object-oriented h-adaptive fluid dynamics simulation. Journal of Computational Physics $215(1), 59-80$.

[37] Straka, J., Wilhelmson, R., Wicker, L., Anderson, J., Doegemeier, K., 1993. Numerical solutions of a nonlinear density current: A benchmark solution and comparison. International J. Num. Methods. Fl. 17, $1-22$. 
[38] Tomita, H., Satoh, M., 2005. A new dynamical framework of non hydrostatic global model using the icosahedral grid. Fluid Dynamics Research $34,357-400$.

[39] Wilcox, L., Giraldo, F. X., Campbell, T., Klockner, A., Warburton, T., Whitcomb, T., 2014. NPS-NRL-Rice-UIUC collaboration on navy atmosphere-ocean coupled models on many-core computer architectures annual report, 1-19.

[40] Yakovlev, S., Moxey, D., Kirby, R. M., Sherwin, S. J., 2015. To cg or to hdg: A comparative study in 3d. Journal of Scientific Computing 67 (1), 192-220.

[41] Zienkiewicz, O. C., 2001. Displacement and equilibrium models in the finite element method by B. Fraeijs de Veubeke, chapter 9, pages 145-197 of stress analysis, edited by O. C. Zienkiewicz and G. S. Holister, published by John Wiley \& Sons, 1965. International Journal for Numerical Methods in Engineering 52 (3), 287-342.

[42] Zienkiewicz, O. C., Taylor, R. L., Too, J. M., 1971. Reduced integration technique in general analysis of plates and shells. International Journal for Numerical Methods in Engineering 3 (2), 275-290. 


\section{A. Constraint imposition example}

Consider a $1 \mathrm{D}$ problem with two linear elements, each $\Delta x=6$ units long. The mass matrix for each element is

$$
\begin{aligned}
& M=\frac{\Delta x}{6}\left[\begin{array}{ll}
2 & 1 \\
1 & 2
\end{array}\right]=\left[\begin{array}{ll}
2 & 1 \\
1 & 2
\end{array}\right] . \\
& \text { (1)-3 (3) }
\end{aligned}
$$

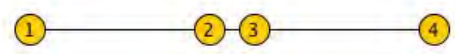

If we use explicit time integration, then all terms but the mass matrix will go to the right hand side. Let us assume the following right hand side vector before we couple the elements using different mechanisms

$$
\left[\begin{array}{llll}
2 & 1 & 0 & 0 \\
1 & 2 & 0 & 0 \\
0 & 0 & 2 & 1 \\
0 & 0 & 1 & 2
\end{array}\right]\left[\begin{array}{l}
\mathbf{q}_{1} \\
\mathbf{q}_{2} \\
\mathbf{q}_{3} \\
\mathbf{q}_{4}
\end{array}\right]=\left[\begin{array}{l}
4 \\
4 \\
8 \\
8
\end{array}\right]
$$

\section{Lagrange multipliers:}

$$
\left[\begin{array}{llll}
2 & 1 & 0 & 0 \\
1 & 2 & 0 & 0 \\
0 & 0 & 2 & 1 \\
0 & 0 & 1 & 2
\end{array}\right]\left[\begin{array}{l}
\mathbf{q}_{\mathbf{1}} \\
\mathbf{q}_{\mathbf{2}} \\
\mathbf{q}_{\mathbf{3}} \\
\mathbf{q}_{\mathbf{4}}
\end{array}\right]=\left[\begin{array}{c}
4 \\
4+\lambda \\
8-\lambda \\
8
\end{array}\right]
$$

or bordering the stiffness matrix with $\mathbf{C}=\left[\begin{array}{llll}0 & -1 & 1 & 0\end{array}\right]$ and $\mathbf{C}^{\top}$

$$
\left[\begin{array}{ccccc}
2 & 1 & 0 & 0 & 0 \\
1 & 2 & 0 & 0 & -1 \\
0 & 0 & 2 & 1 & 1 \\
0 & 0 & 1 & 2 & 0 \\
0 & -1 & 1 & 0 & 0
\end{array}\right]\left[\begin{array}{c}
\mathbf{q}_{1} \\
\mathbf{q}_{2} \\
\mathbf{q}_{3} \\
\mathbf{q}_{4} \\
\lambda
\end{array}\right]=\left[\begin{array}{l}
4 \\
4 \\
8 \\
8 \\
0
\end{array}\right]
$$

which gives the solution vector

$$
\lambda=1 \text { and } \mathbf{q}=\left[\begin{array}{l}
1 \\
2 \\
2 \\
3
\end{array}\right] .
$$

\section{Interior penalties:}

$$
\left[\begin{array}{cccc}
2 & 1 & 0 & 0 \\
1 & 2+w & -w & 0 \\
0 & -w & 2+w & 1 \\
0 & 0 & 1 & 2
\end{array}\right]\left[\begin{array}{l}
\mathbf{q}_{\mathbf{1}} \\
\mathbf{q}_{2} \\
\mathbf{q}_{\mathbf{3}} \\
\mathbf{q}_{4}
\end{array}\right]=\left[\begin{array}{l}
4 \\
4 \\
8 \\
8
\end{array}\right]
$$


Solving the system with different penalty weights $\mathrm{w}=1,100,10000$, we get

$$
\mathbf{q}^{\mathbf{w}=\mathbf{1}}=\left[\begin{array}{c}
1.1429 \\
1.7143 \\
2.2857 \\
2.8571
\end{array}\right], \mathbf{q}^{\mathbf{w}=\mathbf{1 0 0}}=\left[\begin{array}{c}
1.0025 \\
1.9950 \\
2.0050 \\
2.9975
\end{array}\right], \mathbf{q}^{\mathbf{w}=\mathbf{1 0 0 0 0}}=\left[\begin{array}{l}
1 \\
2 \\
2 \\
3
\end{array}\right] .
$$

\section{Master-slave method:}

Using the transformation

$$
\begin{gathered}
\mathbf{q}_{\mathbf{d g}}=\mathbf{T} \mathbf{q}_{\mathbf{c g}} \\
{\left[\begin{array}{l}
\mathbf{q}_{\mathbf{1}} \\
\mathbf{q}_{2} \\
\mathbf{q}_{3} \\
\mathbf{q}_{4}
\end{array}\right]=\left[\begin{array}{lll}
1 & 0 & 0 \\
0 & 1 & 0 \\
0 & 1 & 0 \\
0 & 0 & 1
\end{array}\right]\left[\begin{array}{l}
\mathbf{q}_{\mathbf{1}} \\
\mathbf{q}_{2} \\
\mathbf{q}_{4}
\end{array}\right]}
\end{gathered}
$$

and then applying the following congruent transformations

$$
\begin{aligned}
& \mathbf{K}_{\mathbf{c g}}=\mathbf{T}^{\top} \mathbf{K}_{\mathrm{dg}} \mathbf{T} \\
& \mathbf{R}_{\mathbf{c g}}=\mathbf{T}^{\top} \mathbf{R}_{\mathrm{dg}}
\end{aligned}
$$

to eliminate $\mathbf{q}_{\mathbf{3}}$, we get

$$
\left[\begin{array}{ccc}
2 & 1 & 0 \\
1 & 2+2 & 1 \\
0 & 1 & 2
\end{array}\right]\left[\begin{array}{l}
\mathbf{q}_{1} \\
\mathbf{q}_{2} \\
\mathbf{q}_{4}
\end{array}\right]=\left[\begin{array}{c}
4 \\
4+8 \\
8
\end{array}\right]
$$

which gives the solution vector

$$
\mathbf{q}=\left[\begin{array}{l}
1 \\
2 \\
2 \\
3
\end{array}\right]
$$

CGc:

We can achieve the same result as the master-slave elimination method by using a global storage scheme that does not have node 3 from the beginning. The assembled matrix can be written as follows

$$
\left[\begin{array}{ccc}
2 & 1 & 0 \\
1 & 2+\mathbf{2} & \mathbf{1} \\
0 & \mathbf{1} & \mathbf{2}
\end{array}\right]\left[\begin{array}{l}
\mathbf{q}_{\mathbf{1}} \\
\mathbf{q}_{\mathbf{2}} \\
\mathbf{q}_{\mathbf{4}}
\end{array}\right]=\left[\begin{array}{c}
4 \\
4+\mathbf{8} \\
\mathbf{8}
\end{array}\right]
$$

where bold face values represent contributions from the second element.

CGd:

When we have off-diagonal coefficients, the DSS operation gathers and scatters those coefficients as well. To prevent linear dependence, for instance of rows 
2 and 3 in the following example, we consider the node physically present in an element as master and set the coefficients of the slave nodes to 0 as

$$
\left[\begin{array}{cccc}
2 & 1 & 0 & 0 \\
1 & 2+\mathbf{2} & 0 & \mathbf{1} \\
\mathbf{1} & 0 & \mathbf{2}+2 & 1 \\
0 & 0 & 1 & 2
\end{array}\right]\left[\begin{array}{l}
\mathbf{q}_{1} \\
\mathbf{q}_{2} \\
\mathbf{q}_{3} \\
\mathbf{q}_{4}
\end{array}\right]=\left[\begin{array}{c}
4 \\
4+8 \\
8+4 \\
8
\end{array}\right]
$$

which gives the solution vector

$$
\mathbf{q}=\left[\begin{array}{l}
1 \\
2 \\
2 \\
3
\end{array}\right]
$$

